Johnson \& Wales University ScholarsArchive@JWU

Health \& Wellness Department Faculty

Publications and Research

College of Health \& Wellness

2014

\title{
Surveying Sexual and Gender Minorities
}

Melissa A. Clark

Brown University

Samantha R. Rosenthal

Johnson \& Wales University - Providence, Samantha.Rosenthal@jwu.edu

Ulrike Boehmer

Brown University

Follow this and additional works at: https://scholarsarchive.jwu.edu/health_fac

Part of the Medicine and Health Sciences Commons

\section{Repository Citation}

Clark, Melissa A.; Rosenthal, Samantha R.; and Boehmer, Ulrike, "Surveying Sexual and Gender Minorities" (2014). Health \& Wellness Department Faculty Publications and Research. 23.

https://scholarsarchive.jwu.edu/health_fac/23

This Book Chapter is brought to you for free and open access by the College of Health \& Wellness at ScholarsArchive@JWU. It has been accepted for inclusion in Health \& Wellness Department Faculty Publications and Research by an authorized administrator of ScholarsArchive@JWU. For more information, please contact jcastel@jwu.edu. 

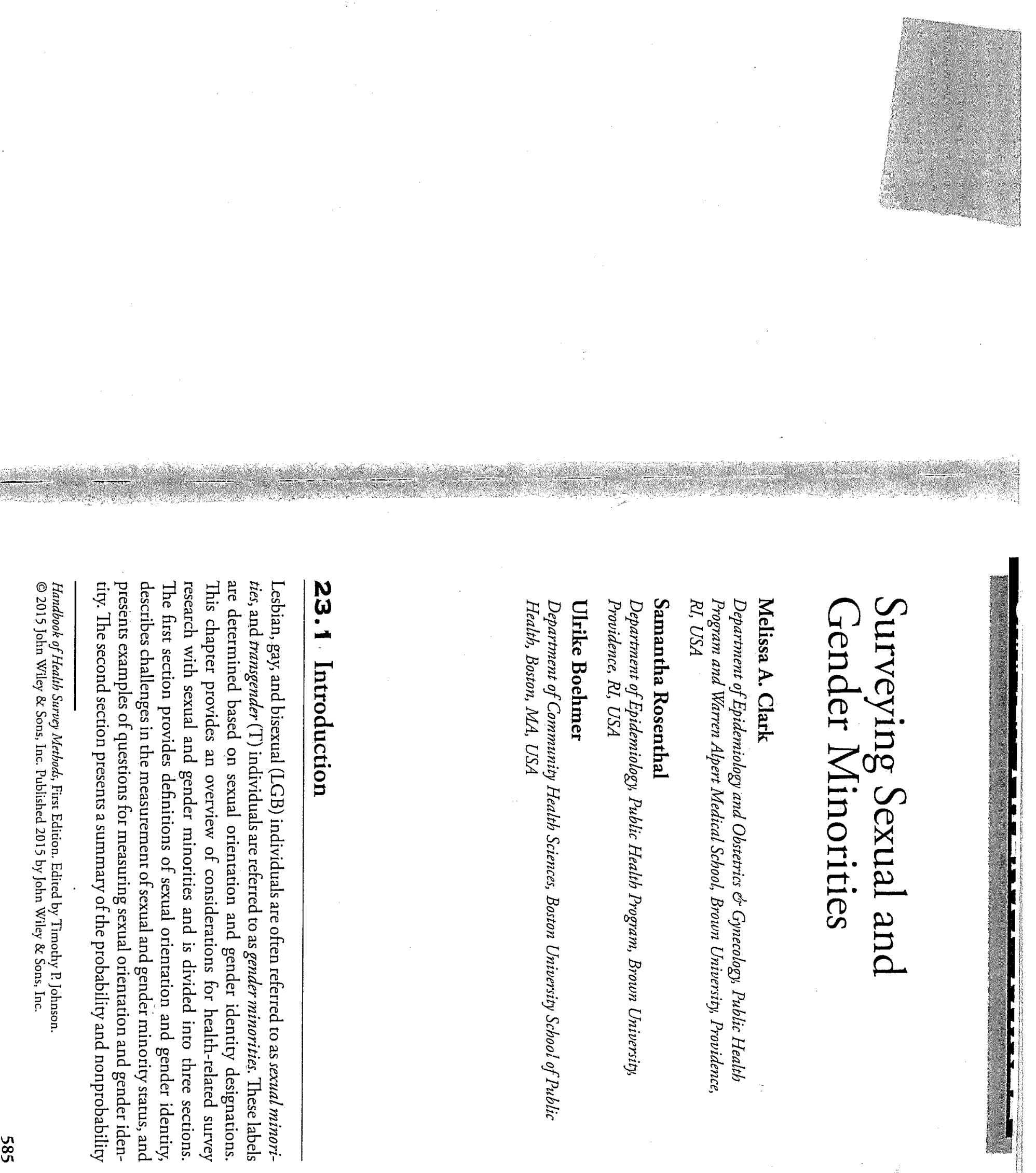


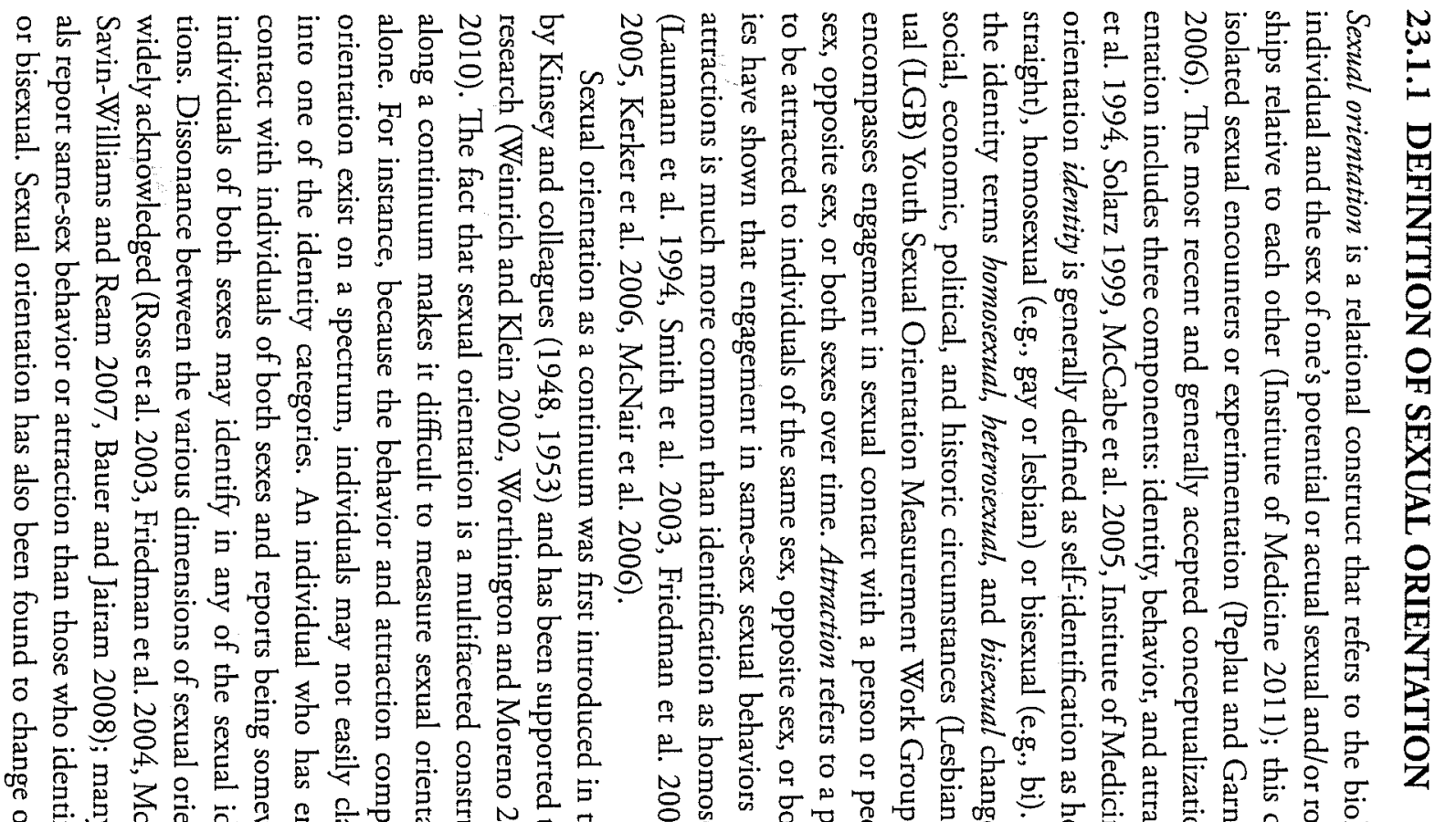

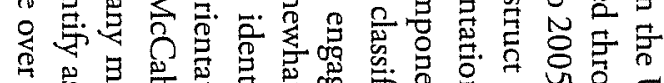

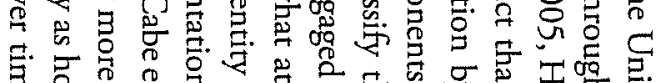

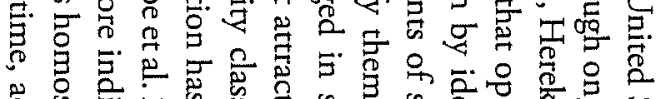

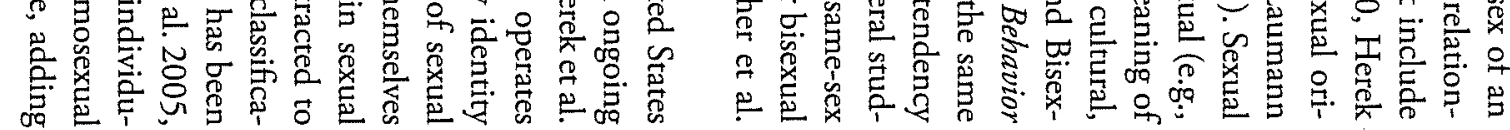
No

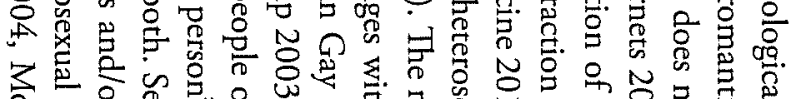

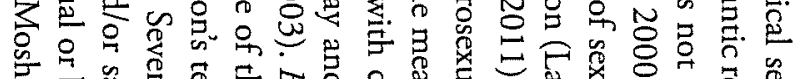

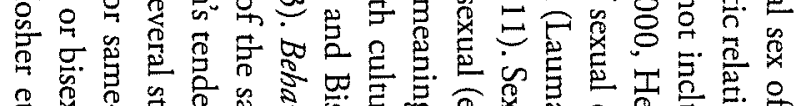

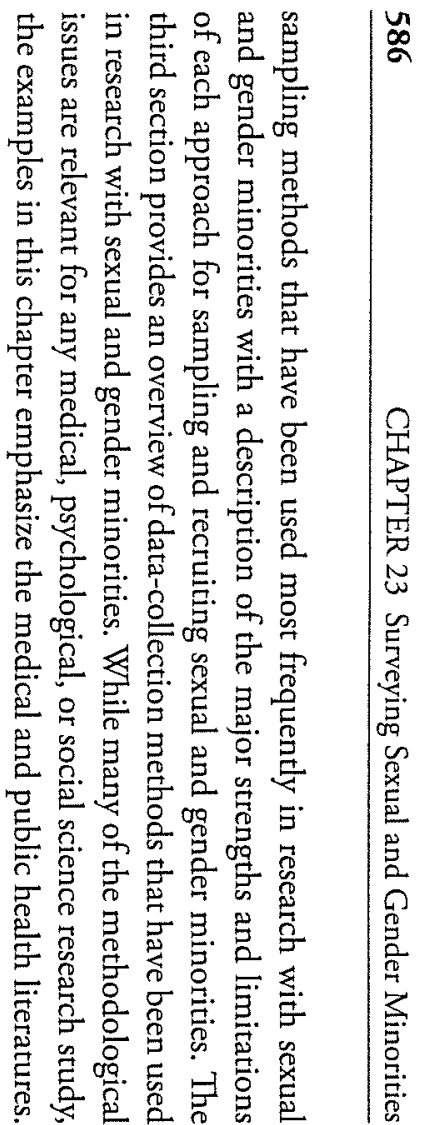

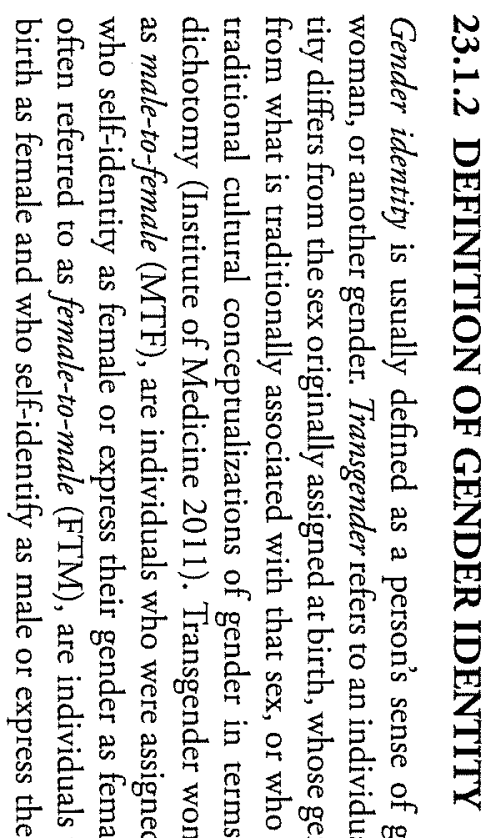

䔽.

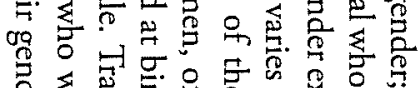

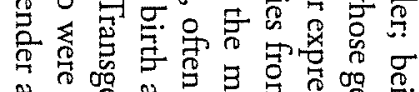

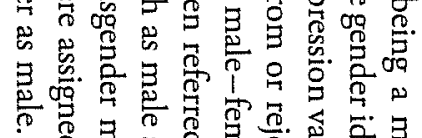

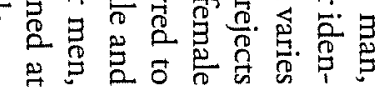

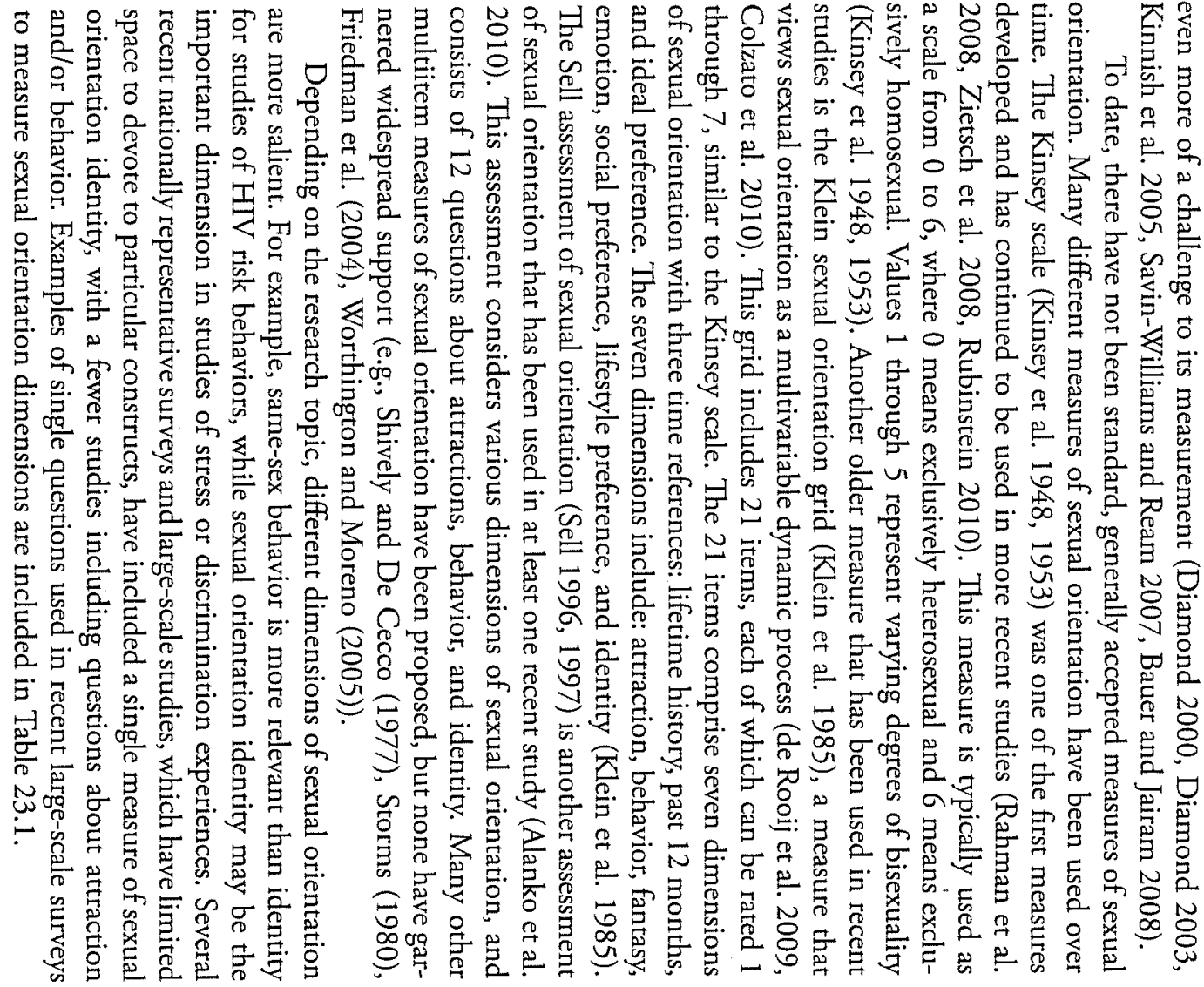


TABLE 23.1 Examples of Sexual Orientation and Gender Identity Questions Used in State and National Surveys by Dimension

\begin{tabular}{|c|c|c|}
\hline Dimension & Question & Example Survey \\
\hline \multirow[t]{3}{*}{$\begin{array}{l}\text { Sexual } \\
\quad \text { orientation-identity }\end{array}$} & $\begin{array}{l}\text { Do you consider yourself to be: } \\
\text { [ ] Heterosexual or straight, } \\
\text { [ ] Gay or lesbian, or } \\
\text { [] Bisexual? }\end{array}$ & $\begin{array}{l}\text { Behavioral Risk Factor Surveillance Survey (state of Vermont) } \\
\text { (www2a.cdc.gov/nccdphp/brfss2/ } \\
\text { coordinator.asp) } \\
\text { HIV/AIDS Surveillance System (www.cdc.gov/hiv/stats/ } \\
\text { hasrlink.HTM) } \\
\text { National Survey of Family Growth }{ }^{a} \text { (www.cdc.gov/nchs/ } \\
\text { nsfg.htm) } \\
\text { National Epidemiologic Survey on Alcohol and Related } \\
\text { Conditions }{ }^{b} \text { (http://www.niaaa.nih.gov/Resources/ } \\
\text { DatabaseResources/Pages/default.aspx) } \\
\text { California Health Interview Survey }{ }^{c} \text { (www.chis.ucla.edu/) }\end{array}$ \\
\hline & $\begin{array}{l}\text { Which of the following best describes you? } \\
\text { [] Heterosexual (straight) } \\
\text { [] Gay or lesbian } \\
\text { [] Bisexual } \\
\text { [] Not sure }\end{array}$ & $\begin{array}{l}\text { Youth Risk Behavior Surveillance Survey (selected states) } \\
\text { (www.cdc.gov/HealthyYouth/yrbs/index.htm) }\end{array}$ \\
\hline & $\begin{array}{l}\text { Do you think of yourself as ... } \\
\text { [ ] Heterosexual or straight (that is, sexually attract } \\
\text { only to [opposite sex]) }\end{array}$ & $\begin{array}{l}\text { National Health and Nutrition Examination Surveyc } \\
\text { (www.cdc.gov/nchs/nhanes.htm) }\end{array}$ \\
\hline
\end{tabular}

[] Homosexual or gay (that is, sexual attracted only

to [same sex])

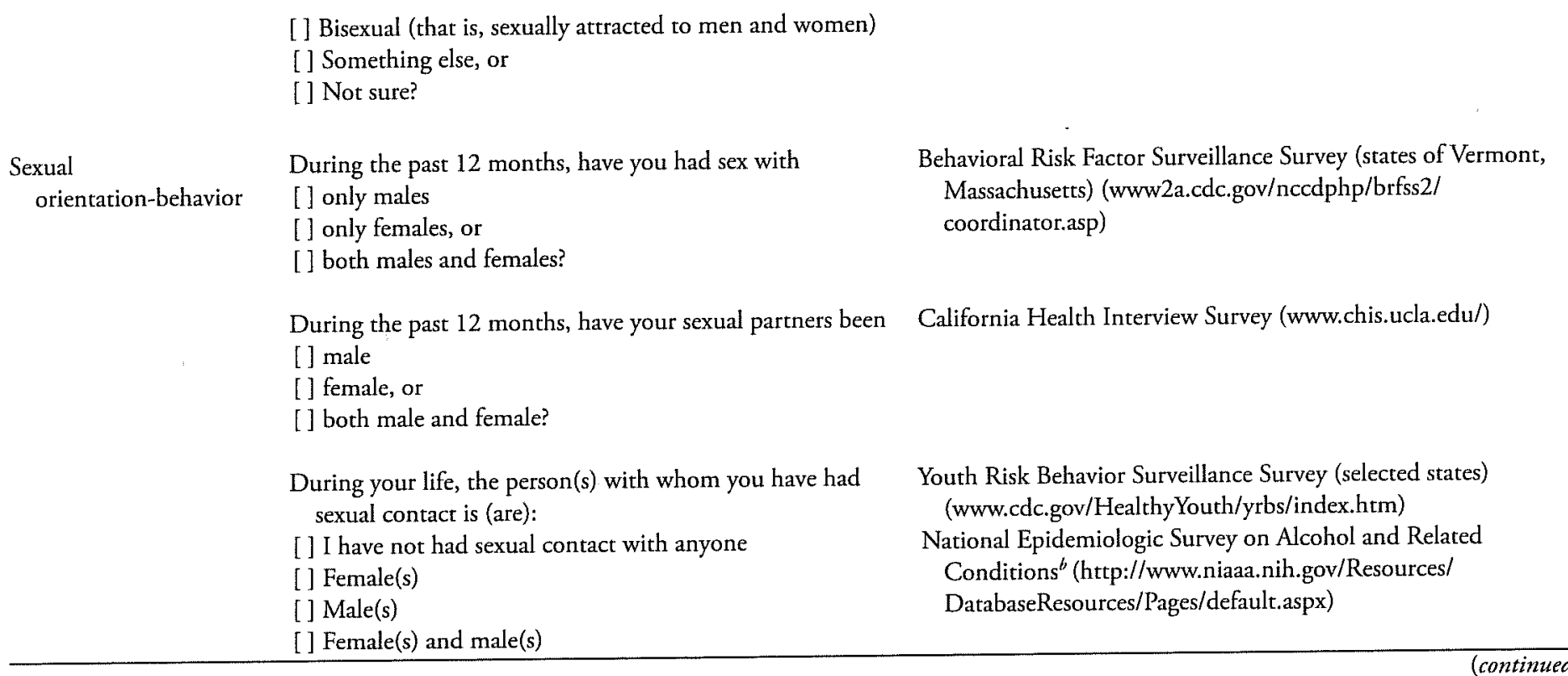




\begin{tabular}{|c|c|c|}
\hline Dimension & Question & Example Survey \\
\hline & $\begin{array}{l}\text { Thinking of the last } 5 \text { years, that is since (season) } \\
\text { of (year), has the partner or partners in your sexual } \\
\text { relationships been: } \\
\text { [ ] only men } \\
\text { [ ] mostly men } \\
\text { [ ] about the same number of men and women } \\
\text { [ ] mostly women } \\
\text { [ ] only women, or } \\
\text { [ ] have you not had a sexual relationship in the } \\
\text { last five years? }\end{array}$ & National Alcohol Survey (www.arg.org/address.hrml) \\
\hline $\begin{array}{l}\text { Sexual } \\
\quad \text { orientation-attraction }\end{array}$ & $\begin{array}{l}\text { People are different in their sexual attraction to } \\
\text { other people. Which best describes } \\
\text { your feelings? Are you } \\
\text { [] only attracted to females } \\
\text { [ ] mostly attracted to females } \\
\text { [] equally attracted to females and males } \\
\text { [] mostly attracted to males } \\
\text { [ ] only attracted to males, or } \\
\text { [] not sure? }\end{array}$ & $\begin{array}{l}\text { National Survey of Family Growth (www.cdc.gov/nchs/nsfg.htm) } \\
\text { National Epidemiologic Survey on Alcohol and Related } \\
\text { Conditions }{ }^{b} \text { (http://www.niaaa.nih.gov/Resources/ } \\
\text { DatabaseResources/Pages/default.aspx) }\end{array}$ \\
\hline
\end{tabular}

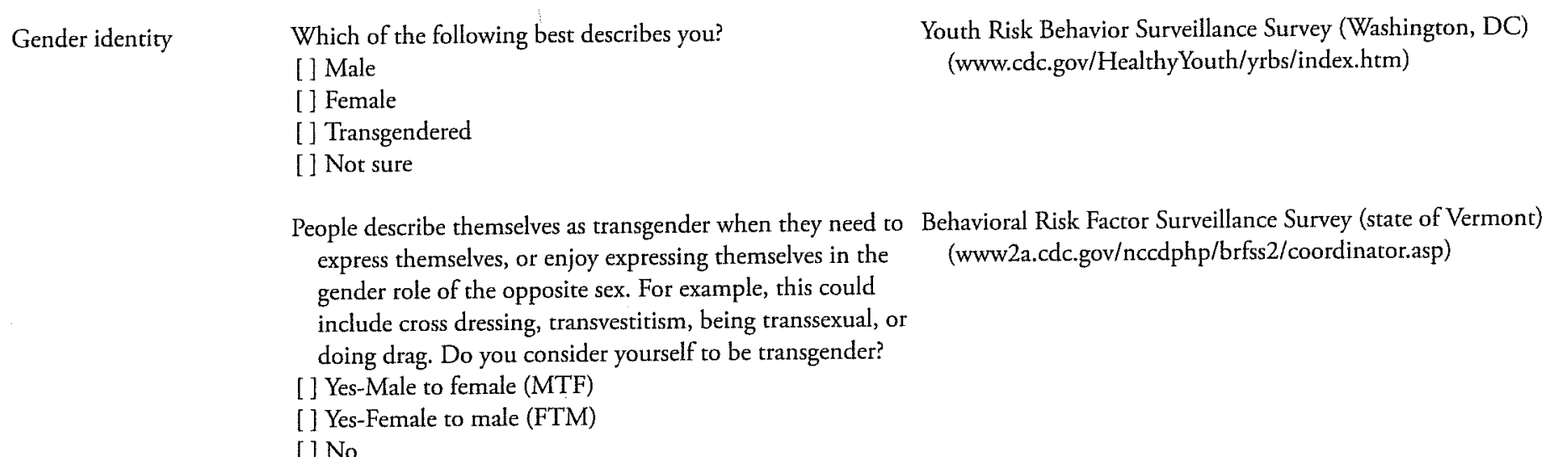

ancludes additional response of "or something else?"

${ }^{b}$ Question wording varies slightly by year of administration.

'Second category phrased gay (lesbian) or homosexual. 

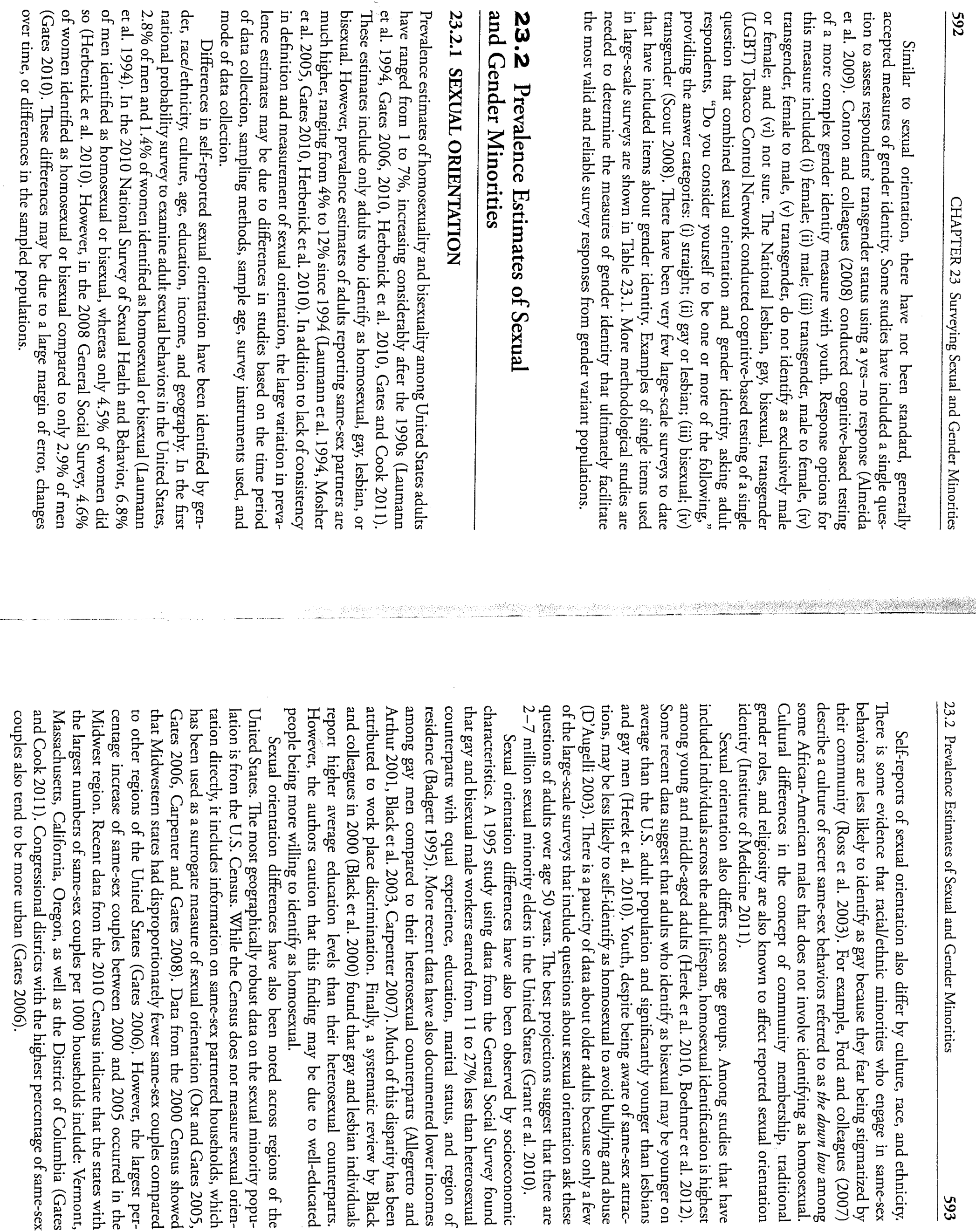

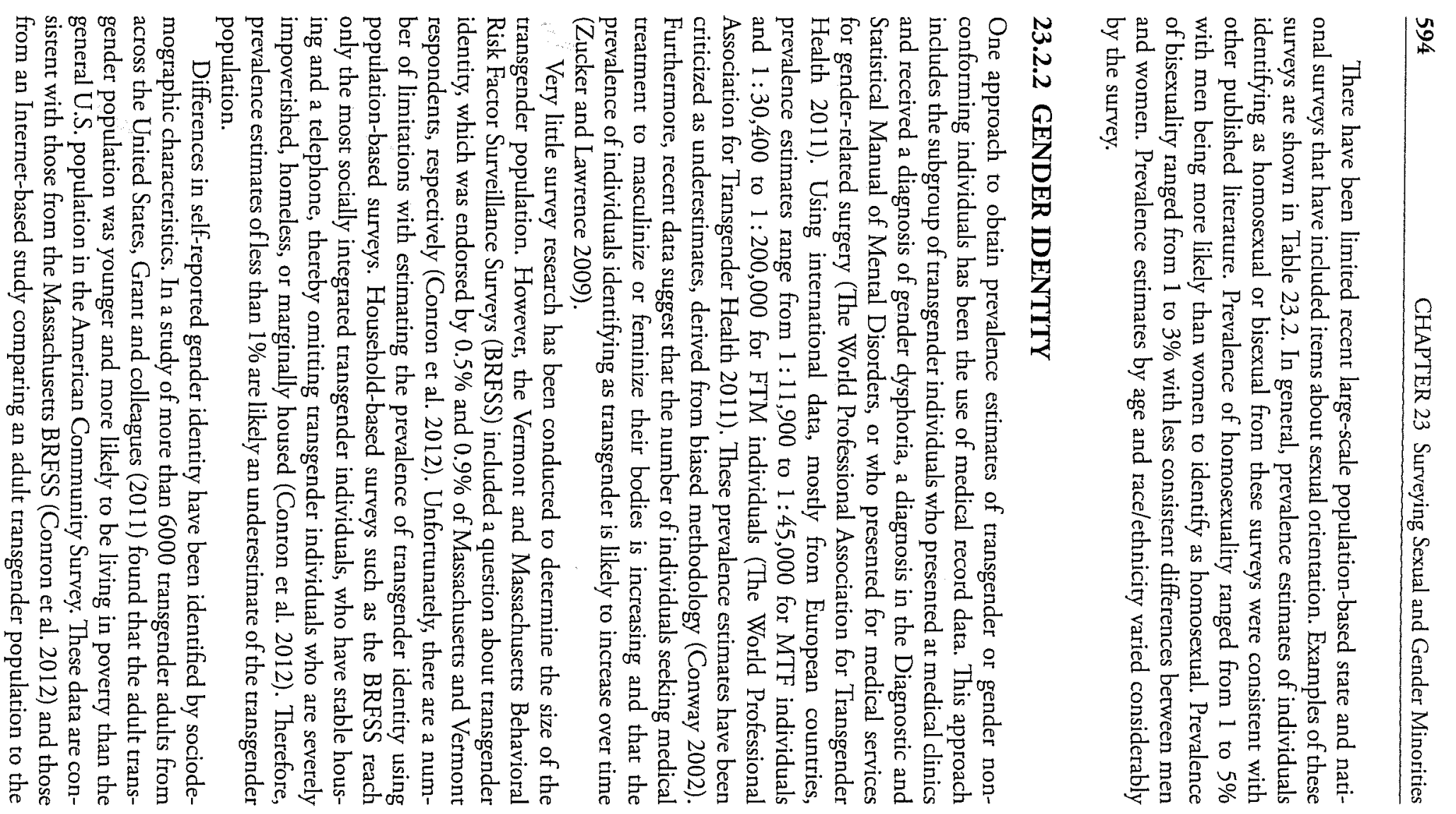

TABLE 23.2 Prevalence Estimates (\%) and Mode of Data Collection for Self-Reported Sexual Orientation Identity from Selected Population-Based Surveys

\begin{tabular}{|c|c|c|c|c|c|c|c|c|c|c|c|c|c|c|c|}
\hline & \multicolumn{3}{|c|}{$\begin{array}{c}\text { General Social } \\
\text { Survey }(2010)^{a} \\
n=2044\end{array}$} & \multicolumn{3}{|c|}{$\begin{array}{c}\text { National Health } \\
\text { and Nutrition } \\
\text { Examination Survey } \\
\begin{array}{c}(2007-2008)^{k, a} \\
n=3265\end{array} \\
\end{array}$} & \multicolumn{3}{|c|}{$\begin{array}{l}\text { National Study } \\
\text { of Family } \\
\text { Growth }(2002)^{c_{1} d} \\
n=12,571\end{array}$} & \multicolumn{3}{|c|}{$\begin{array}{l}\text { California Health } \\
\text { Interview } \\
\text { Survey }(2007)^{a} \\
n=41,157\end{array}$} & \multicolumn{3}{|c|}{$\begin{array}{c}\text { Behavioral Risk } \\
\text { Factor Surveillance } \\
\text { System: Washington, DC } \\
(2005 \text { and } 2007 \\
\text { combined) } n=6218\end{array}$} \\
\hline & Homo & $\mathrm{Bi}$ & Ref & Homo & $\mathrm{Bi}^{f}$ & Ref & Homo & $\mathrm{Bi}^{f}$ & Ref & Homo & $\mathrm{Bi}$ & Ref & Homo & $\mathrm{Bi}^{f}$ & Ref \\
\hline \multicolumn{16}{|c|}{$\begin{array}{l}\text { Prevalence } \\
\text { estimates }\end{array}$} \\
\hline Overall & 1.2 & 1.4 & 1.4 & 1.5 & 2.8 & 0.1 & 1.7 & 2.4 & 1.8 & 2.2 & 1.2 & - & 4.9 & 2.3 & 3.2 \\
\hline $\begin{array}{l}\text { Gender } \\
\text { Male }\end{array}$ & 1.3 & 0.5 & 0.9 & 1.9 & 1.4 & 0.0 & 2.3 & 1.8 & 1.8 & 3.2 & 1.1 & - & 8.3 & 2.4 & - \\
\hline Female & 1.2 & 2.1 & 1.4 & 1.1 & 4.2 & 0.2 & 1.3 & 2.8 & 1.8 & 1.5 & 1.3 & - & 2.0 & 2.2 & - \\
\hline \multicolumn{16}{|c|}{ Age (years) } \\
\hline $18-24$ & 2.4 & 1.9 & 1.5 & 0.7 & 6.9 & 0.2 & - & - & - & 1.5 & 2.2 & - & 3.7 & 3.7 & - \\
\hline $25-34$ & 0.9 & 2.6 & 0.8 & 2.1 & 3.2 & 0.2 & - & - & - & 1.7 & 1.8 & - & 5.7 & 1.9 & - \\
\hline $35-44$ & 1.9 & 1.6 & 0.8 & 1.9 & 3.0 & 0.1 & - & - & - & 2.4 & 1.4 & - & 6.2 & 3.7 & - \\
\hline $45-53$ & 1.2 & 1.4 & 0.7 & 1.0 & 1.6 & 0.2 & - & - & - & 2.9 & 1.1 & - & 6.7 & 1.8 & - \\
\hline $55-64$ & 1.4 & 0.8 & 0.8 & 1.3 & 1.3 & 0.0 & - & - & - & 2.1 & 0.9 & - & 4.3 & 0.8 & - \\
\hline$\geq 65$ & 0.0 & 0.0 & 2.9 & - & - & - & - & - & - & 1.4 & 0.9 & - & 1.0 & 1.7 & - \\
\hline \multicolumn{16}{|c|}{ Race/ethnicity } \\
\hline White & 1.4 & 1.4 & 1.2 & 1.1 & 2.8 & 0.0 & - & - & - & 2.6 & 1.3 & - & 9.0 & 2.0 & - \\
\hline
\end{tabular}


TABLE 23.2 (Continued)

\begin{tabular}{|c|c|c|c|c|c|c|c|c|c|c|c|c|c|c|c|}
\hline & \multicolumn{3}{|c|}{$\begin{array}{l}\text { General Social } \\
\text { Survey }(2010)^{a} \\
n=2044\end{array}$} & \multicolumn{3}{|c|}{$\begin{array}{c}\text { National Health } \\
\text { and Nutrition } \\
\text { Examination Survey } \\
(2007-2008)^{b, a} \\
n=3265 \\
\end{array}$} & \multicolumn{3}{|c|}{$\begin{array}{l}\text { National Study } \\
\text { of Family } \\
\text { Growth }(2002)^{c, d} \\
n=12,571\end{array}$} & \multicolumn{3}{|c|}{ 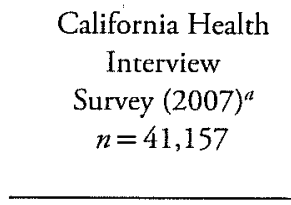 } & \multicolumn{3}{|c|}{$\begin{array}{c}\text { Behavioral Risk } \\
\text { Factor Surveillance } \\
\text { System: Washington, DC } \\
(2005 \text { and } 2007 \\
\text { combined })^{c} n=6218 \\
\end{array}$} \\
\hline & Homo & $\mathrm{Bi}$ & Ref & Homo & $\mathrm{Bi}^{f}$ & Ref & Homo & $\mathrm{Bi}^{f}$ & Ref & Homo & $\mathrm{Bi}$ & Ref & Homo & $\mathrm{Bi}^{f}$ & Ref \\
\hline Black & 0.9 & 1.5 & 1.9 & 2.2 & 4.2 & 0.0 & - & - & - & 2.0 & 1.2 & - & 2.0 & 2.2 & - \\
\hline Hispanic/Latino & - & - & - & 2.1 & 2.4 & 0.8 & - & - & - & 1.3 & 1.0 & - & 5.3 & 4.8 & - \\
\hline Asian & - & - & - & - & - & - & - & - & - & 0.9 & 0.9 & - & - & - & - \\
\hline Other & 0.5 & 1.3 & 0.5 & 2.9 & 1.5 & 0.0 & - & - & - & 2.3 & 1.3 & - & 5.2 & 1.3 & - \\
\hline $\begin{array}{l}\text { Mode of data } \\
\text { collection for } \\
\text { sexual } \\
\text { orientation } \\
\text { questions }\end{array}$ & \multicolumn{3}{|c|}{$\begin{array}{l}\text { Computer-assisted } \\
\text { personal interviews }\end{array}$} & \multicolumn{3}{|c|}{$\begin{array}{l}\text { Audio computer-assisted } \\
\text { self-interviews }\end{array}$} & \multicolumn{3}{|c|}{$\begin{array}{l}\text { Audio computer-assisted } \\
\text { self-interviews }\end{array}$} & \multicolumn{3}{|c|}{$\begin{array}{l}\text { Computer-assisted } \\
\text { telephone interviews }\end{array}$} & \multicolumn{3}{|c|}{$\begin{array}{l}\text { Computer-assisted } \\
\text { telephone interviews }\end{array}$} \\
\hline
\end{tabular}

-, Data not available.

Homo, self-reported homosexual orientation; Bi, seff-reported bisexual orientation; Ref, refused question about sexual orientation. "Analysis computed for this chaprer.

"Includes only ages 20-59.

'Includes only ages $15-44$.

${ }^{d}$ Data from Mosher et al. (2005).

'Data from Dyer er al. (2010).

Bisexual includes individuals reporting "orher"

Includes only ages $18-70$.

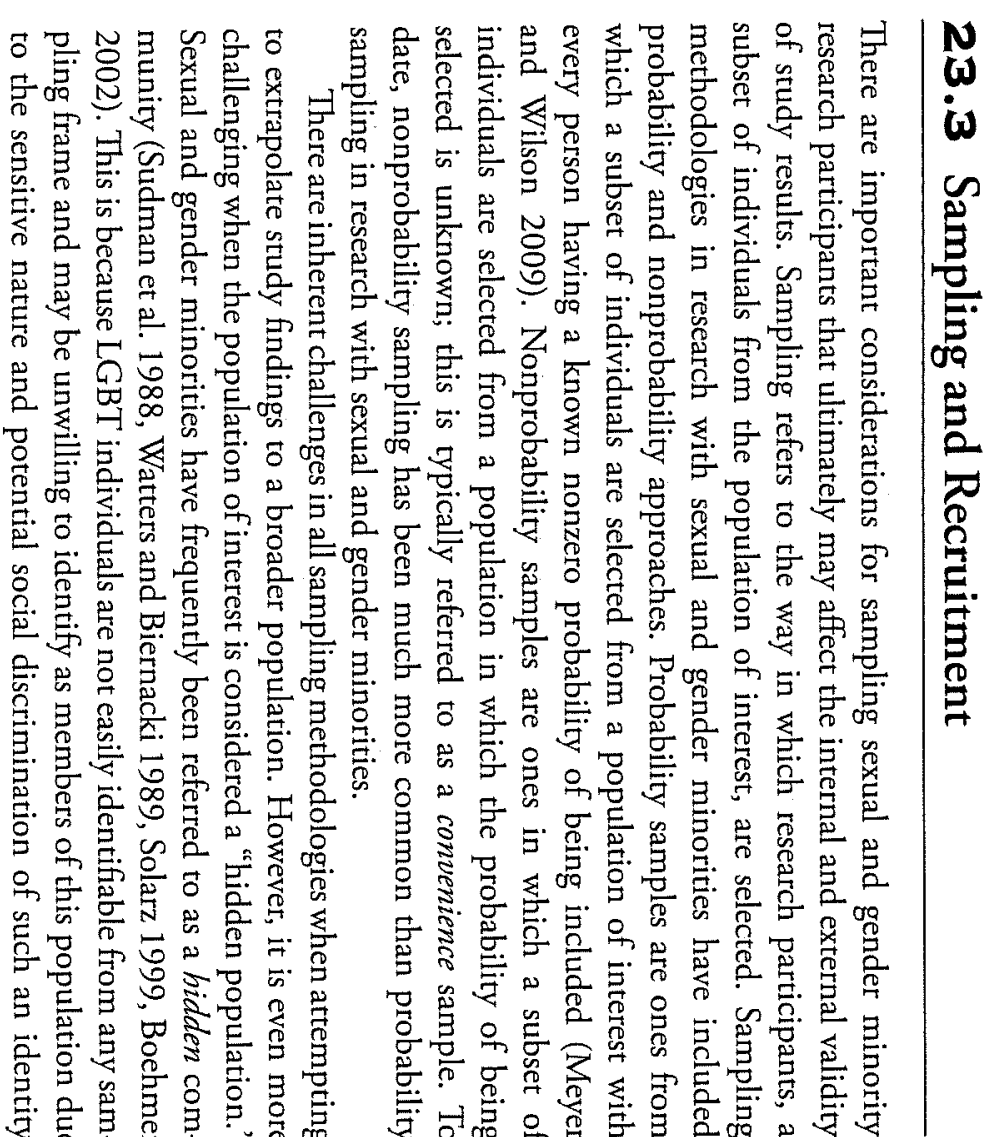

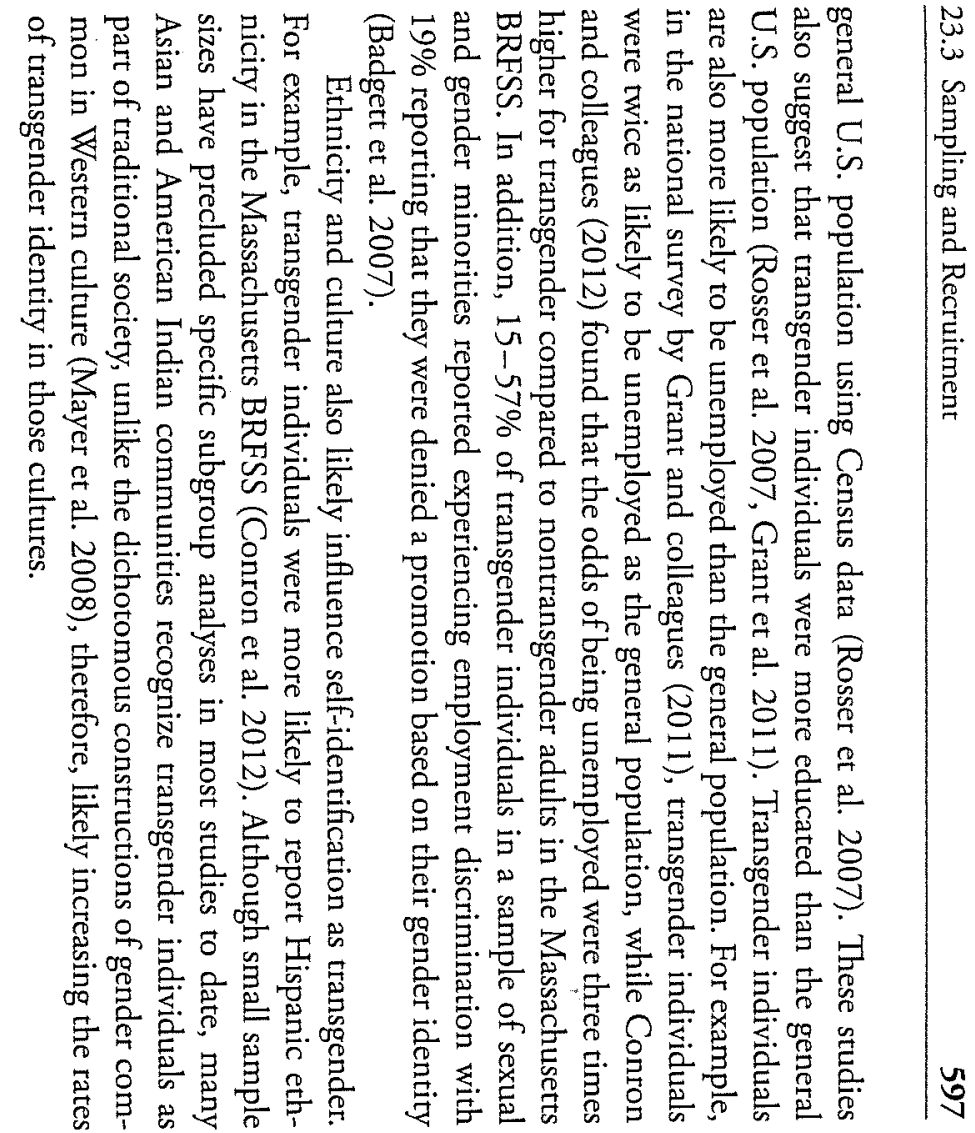



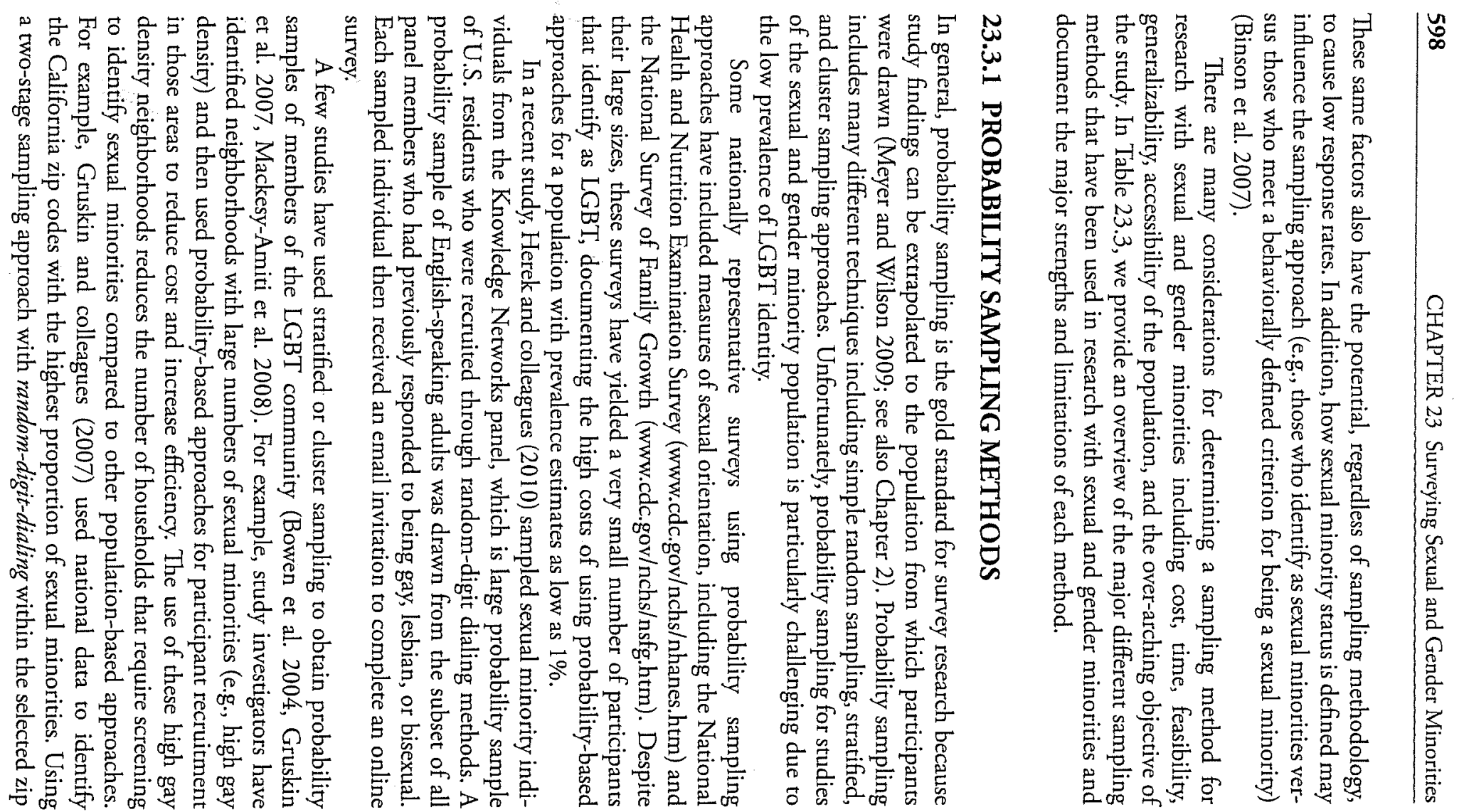

TABLE 23.3 Strengths and Limitations of Sampling Methods Used in Studies with Sexual and Gender Minorities

\begin{tabular}{|c|c|c|c|}
\hline Sampling Method & Strengths & Limitations & Example Studies thar have Used Method \\
\hline \multicolumn{4}{|l|}{ Probability sampling } \\
\hline Random-digit dialing & $\begin{array}{l}\text { Estimates can be } \\
\text { extrapolared to the } \\
\text { population from which } \\
\text { study subjects were } \\
\text { drawn }\end{array}$ & $\begin{array}{l}\text { High costs due to low prevalence } \\
\text { of sexual and gender minorities } \\
\text { Low coverage of individuals with } \\
\text { no or sporadic telephone } \\
\text { availability }\end{array}$ & $\begin{array}{l}\text { (Gruskin et al. (2007), Herek } \\
\text { et al. (2010) }\end{array}$ \\
\hline Household-based sampling & $\begin{array}{l}\text { Estimates can be } \\
\text { extrapolated to the } \\
\text { population from which } \\
\text { study subjects were } \\
\text { drawn }\end{array}$ & $\begin{array}{l}\text { High costs due to low prevalence } \\
\text { of sexual minorities } \\
\text { Potential bias toward individuals } \\
\text { living in areas with higher } \\
\text { densities of sexual minorities }\end{array}$ & Bowen et al. (2004) \\
\hline \multicolumn{4}{|l|}{ Nonprobability sampling } \\
\hline List-based sampling & $\begin{array}{l}\text { Provides an easy and } \\
\text { accessible sampling } \\
\text { frame }\end{array}$ & $\begin{array}{l}\text { Can only generalize findings to } \\
\text { others in the list population } \\
\text { Lists of sexual and gender } \\
\text { minorities are generally } \\
\text { unavailable } \\
\text { Quality of available lists must be } \\
\text { carefully assessed for incorrect, } \\
\text { incomplete, and duplicate } \\
\text { information }\end{array}$ & Solomon et al. (2004) \\
\hline
\end{tabular}


ま

\begin{tabular}{|c|c|c|c|}
\hline Sampling Method & Strengths & Limitations & Example Studies that have Used Method \\
\hline Time-location sampling & $\begin{array}{l}\text { Can help to increase sample } \\
\text { size when recruiting } \\
\text { sexual minorities } \\
\text { If all relevant venues are } \\
\text { included and all } \\
\text { segments of population } \\
\text { visit these venues, this } \\
\text { can be considered a } \\
\text { probability sampling }\end{array}$ & $\begin{array}{l}\text { Appropriate venues for } \\
\text { recruitment may change over } \\
\text { time and must be reevaluated } \\
\text { continually } \\
\text { Venues and times selected for data } \\
\text { collection may not include } \\
\text { certain segments of the } \\
\text { population } \\
\text { Nonresponse may be a problem at } \\
\text { stigmatized venues }\end{array}$ & $\begin{array}{l}\text { MacKellar et al. (1996), Muhib } \\
\text { et al. (2001), Cai et al. (2010) }\end{array}$ \\
\hline Snowball sampling & $\begin{array}{l}\text { Can help to increase sample } \\
\text { size when recruiting } \\
\text { sexual minorities } \\
\text { May be able to better } \\
\text { recruit hard-to-reach } \\
\text { individuals not enrolled } \\
\text { through other sampling } \\
\text { schemes }\end{array}$ & $\begin{array}{l}\text { Members of the same social } \\
\text { network are likely to be more } \\
\text { socially connected and thus } \\
\text { more similar than the broader } \\
\text { sexual and gender minority } \\
\text { population }\end{array}$ & $\begin{array}{l}\text { Warner et al. (2004), Browne } \\
\text { (2005), Kendall et al. (2008), } \\
\text { Balsam et al. (2010), Feng et al. } \\
\text { (2010), Lehavot and Simoni } \\
\text { (2011), Prado Cortez et al. } \\
\text { (2011). }\end{array}$ \\
\hline Respondent-driven sampling & $\begin{array}{l}\text { Can help to increase sample } \\
\text { size when recruiting } \\
\text { sexual minorities }\end{array}$ & $\begin{array}{l}\text { Assumptions of the methodology } \\
\text { must be met }\end{array}$ & $\begin{array}{l}\text { Ramirez-Valles et al. (2005), } \\
\text { Johnston et al. (2008), Kendall } \\
\text { et al. (2008), Lauby et al. }\end{array}$ \\
\hline
\end{tabular}

\begin{tabular}{|c|c|c|c|}
\hline & $\begin{array}{l}\text { Associated with lower costs } \\
\text { than time-location } \\
\text { sampling } \\
\text { Can be used to derive } \\
\text { valid, unbiased } \\
\text { population estimates }\end{array}$ & $\begin{array}{l}\text { May not be able to reach an } \\
\text { adequate sampling size in } \\
\text { subgroups of sexual and gender } \\
\text { minority population with } \\
\text { limited social connections }\end{array}$ & $\begin{array}{l}\text { (2008), Ramirez-Valles et al. } \\
\text { (2008) Richards et al. (2008), } \\
\text { Wheeler et al. (2008), Evans } \\
\text { et al. (2011) }\end{array}$ \\
\hline Web-based sampling & $\begin{array}{l}\text { population estimates } \\
\text { Can help to increase sample } \\
\text { size when recruiting } \\
\text { sexual minorities } \\
\text { May be particularly }\end{array}$ & $\begin{array}{l}\text { Samples are not likely } \\
\text { representative of the broader } \\
\text { sexual and gender minority } \\
\text { population }\end{array}$ & $\begin{array}{l}\text { Rosser et al. (2007), Johnston } \\
\text { et al. (2008), Chesir-Teran and } \\
\text { Hughes (2009), Evans et al. } \\
(2011)\end{array}$ \\
\hline Advertising & $\begin{array}{l}\text { May be particularly } \\
\text { effective for recruiting } \\
\text { younger populations } \\
\text { Can help to increase sample } \\
\text { size when recruiting } \\
\text { sexual minorities }\end{array}$ & $\begin{array}{l}\text { Samples are not likely } \\
\text { representative of the broader } \\
\text { sexual and gender minority } \\
\text { population }\end{array}$ & $\begin{array}{l}\text { Silvestre et al. (2006), } \\
\text { Chesir-Teran and Hughes } \\
\text { (2009) }\end{array}$ \\
\hline
\end{tabular}




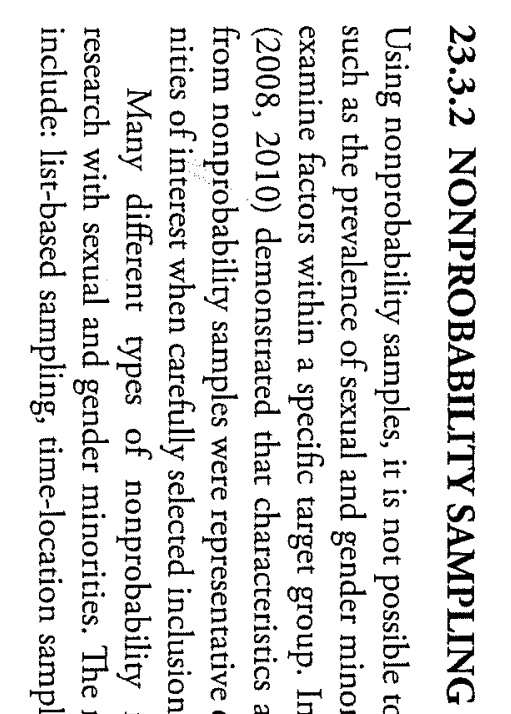

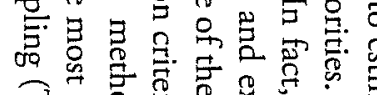

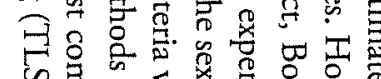

cos

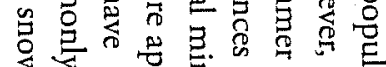

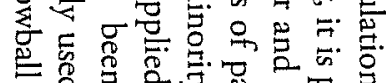

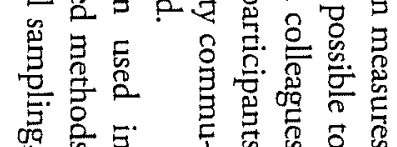

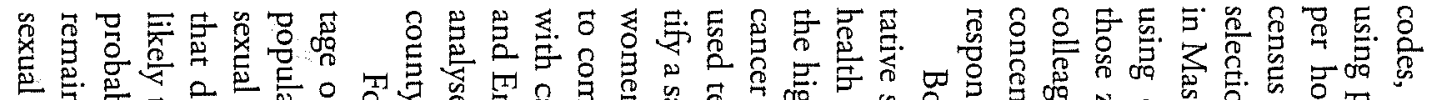

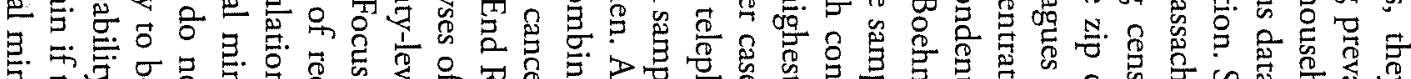

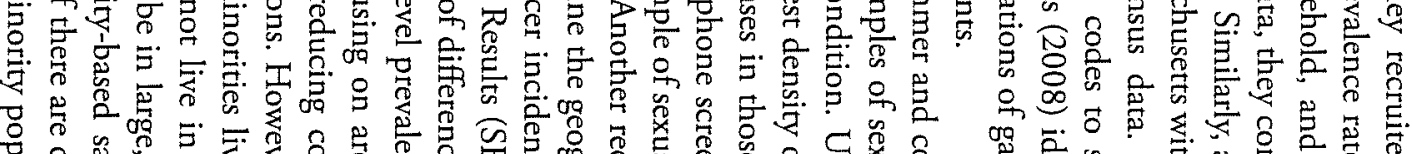

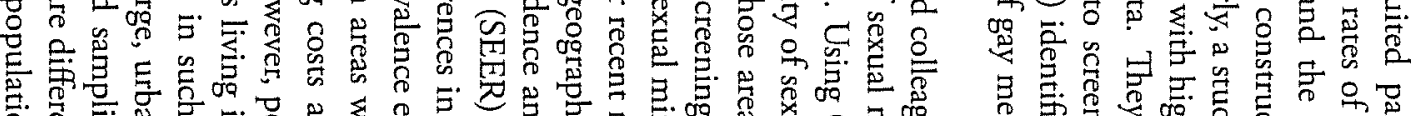

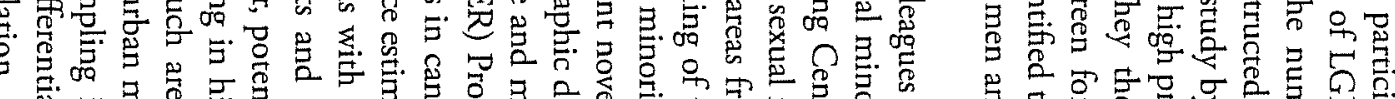

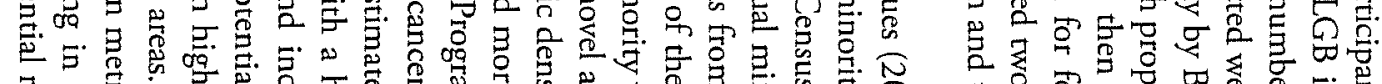

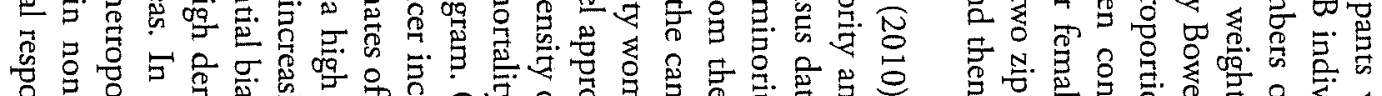

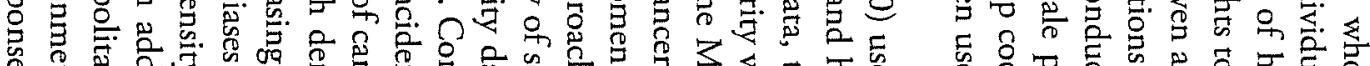

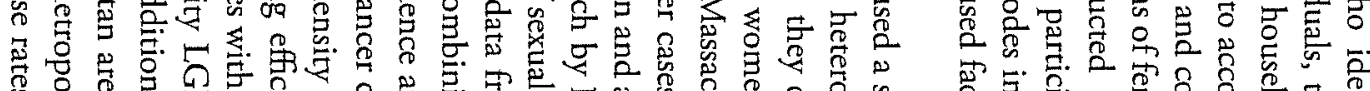

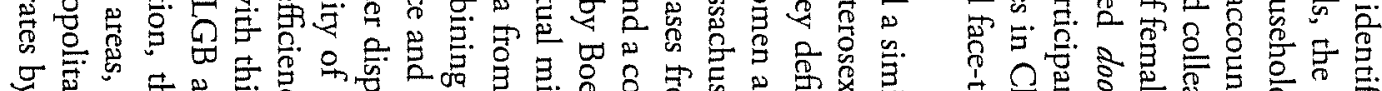

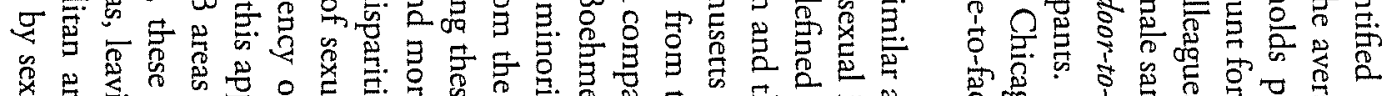

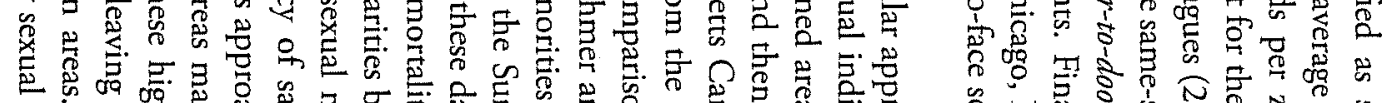

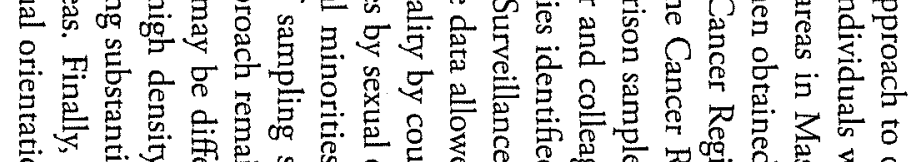

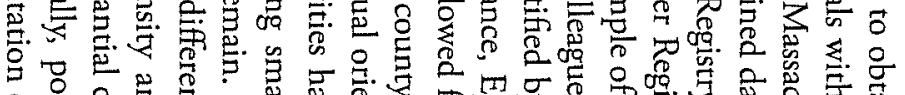

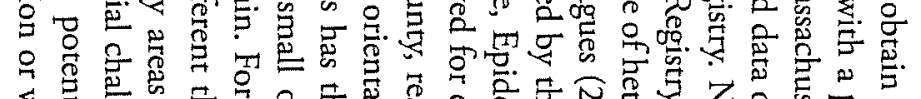

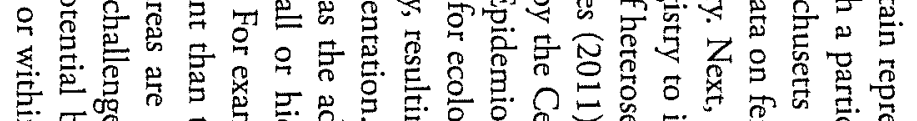

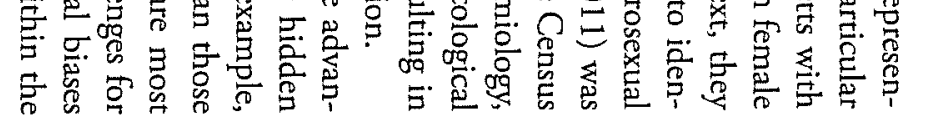

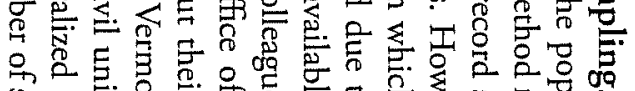

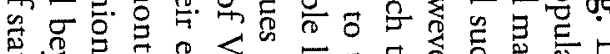

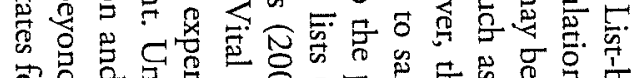

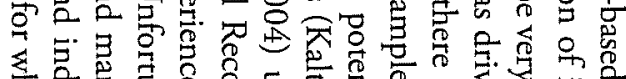

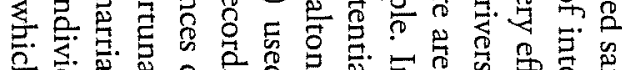

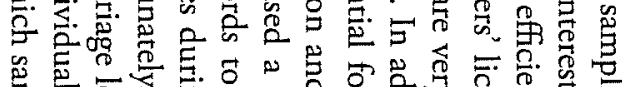

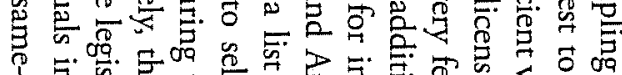

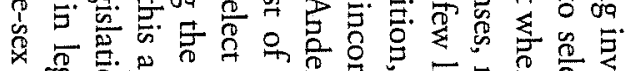

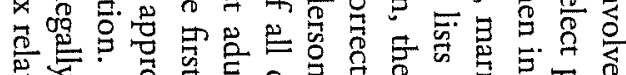

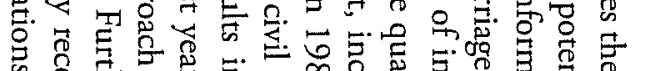

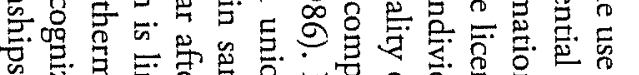

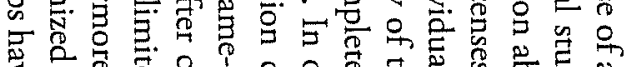
等

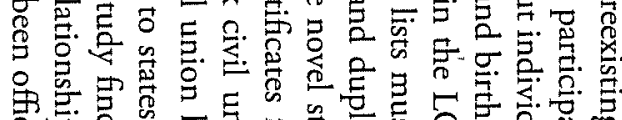

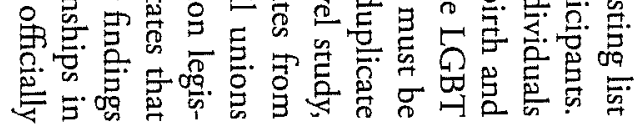




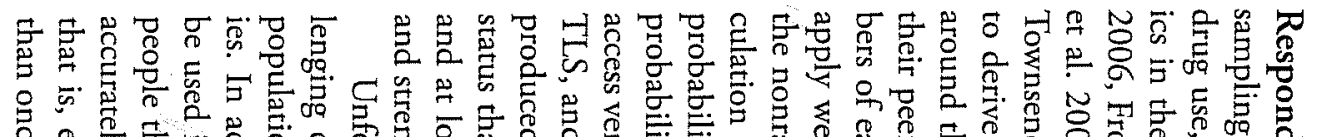

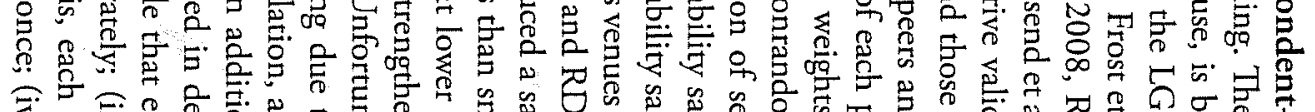

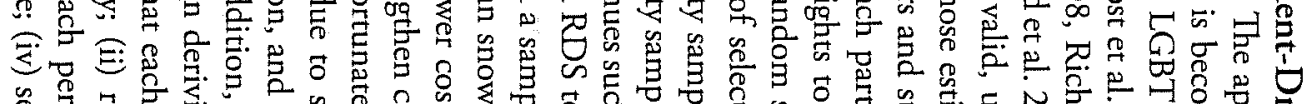

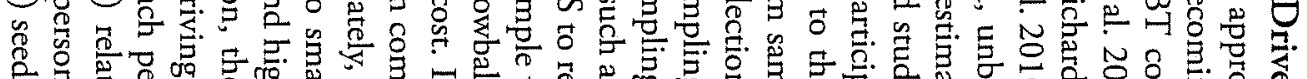

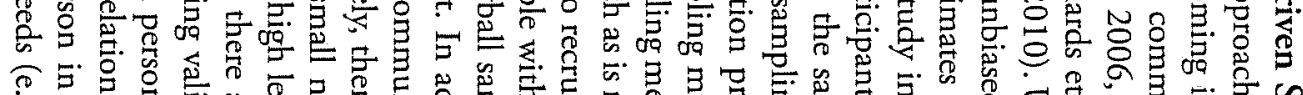

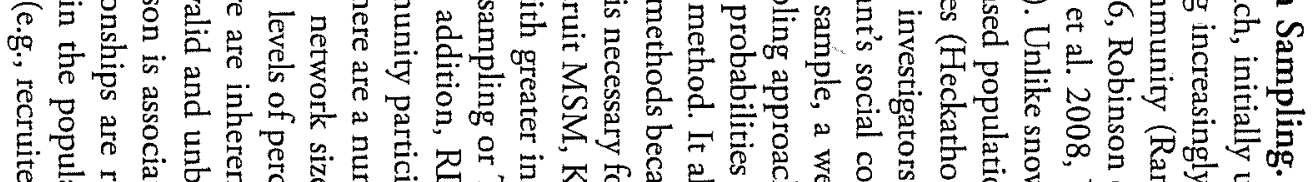

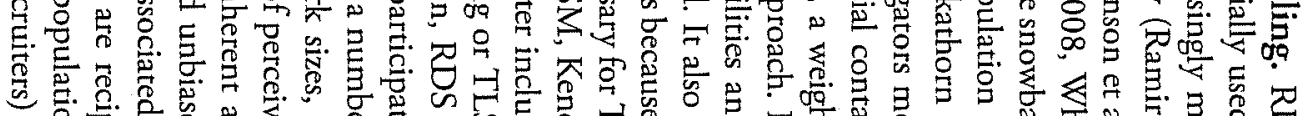

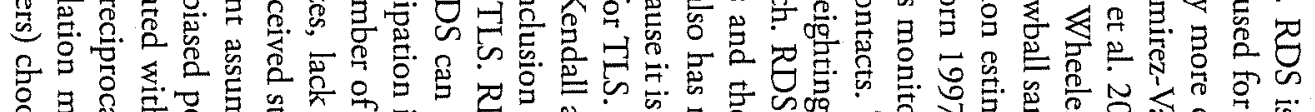

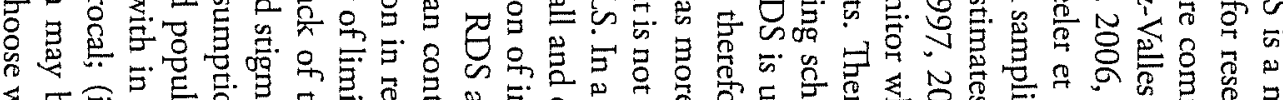

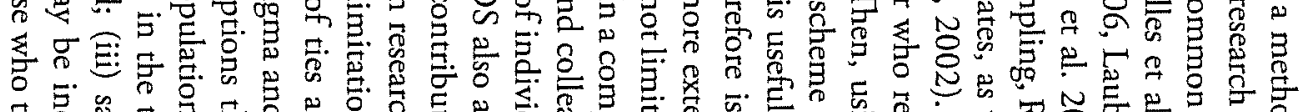

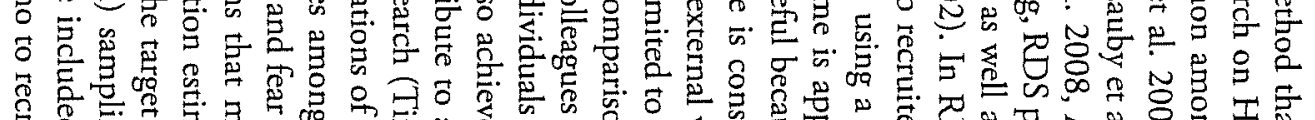

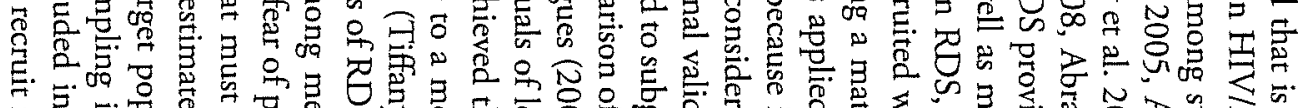

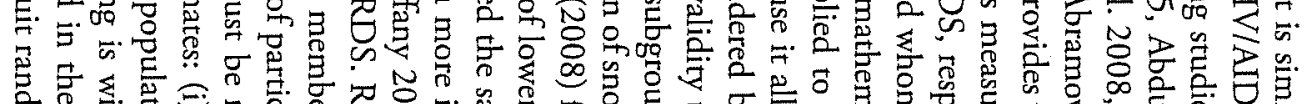

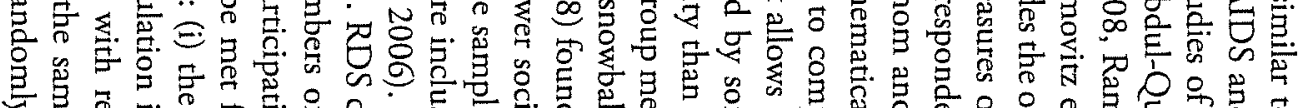

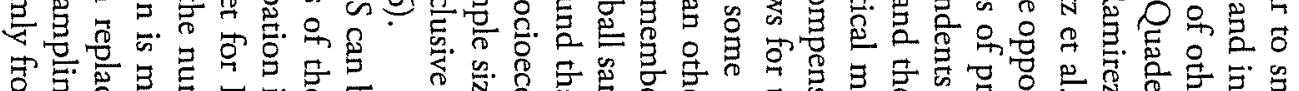

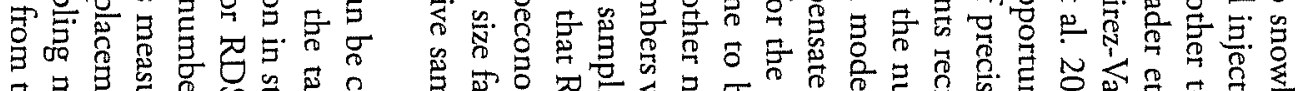

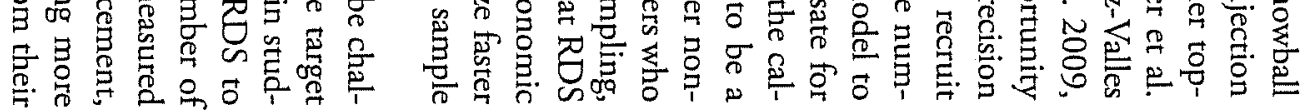
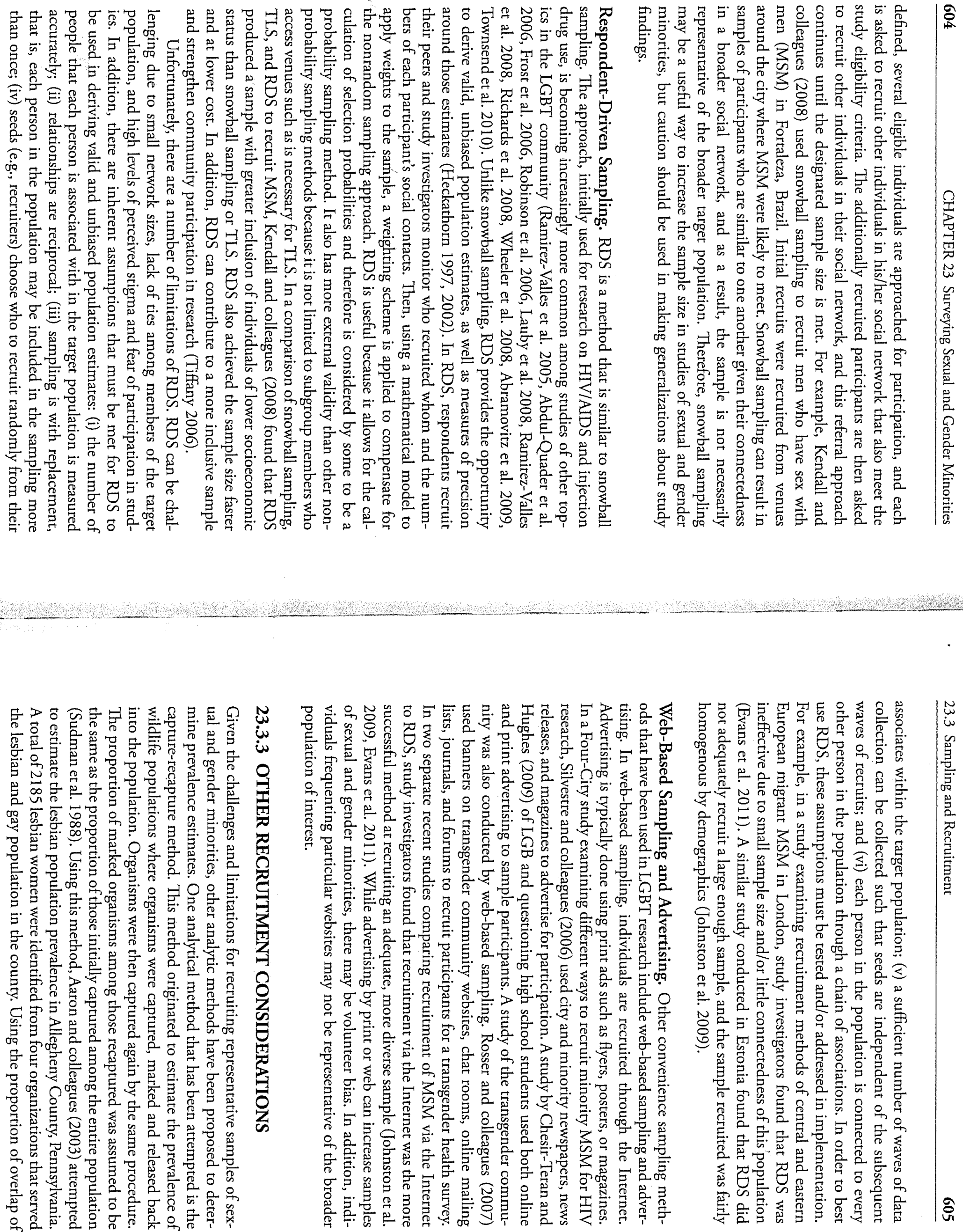


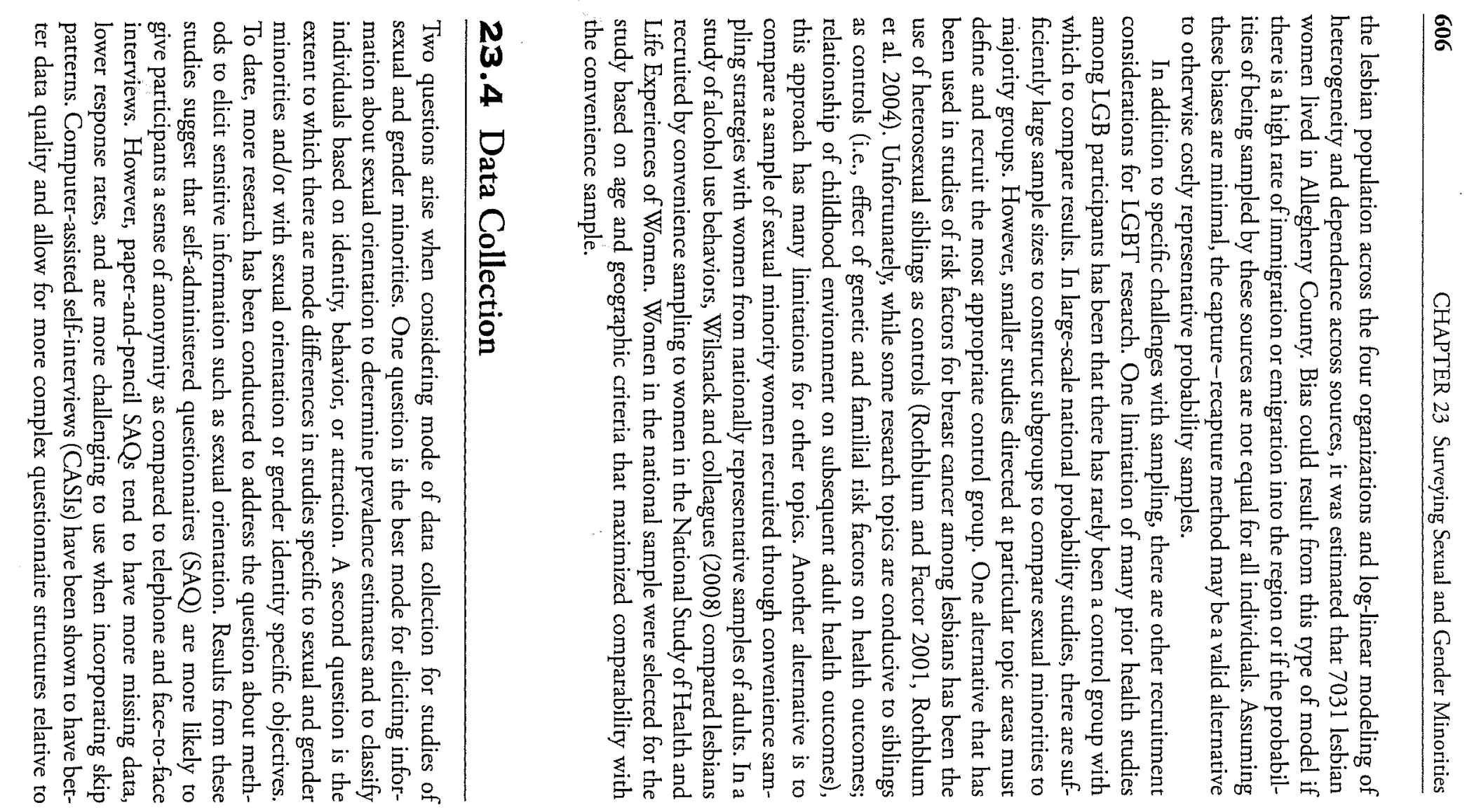

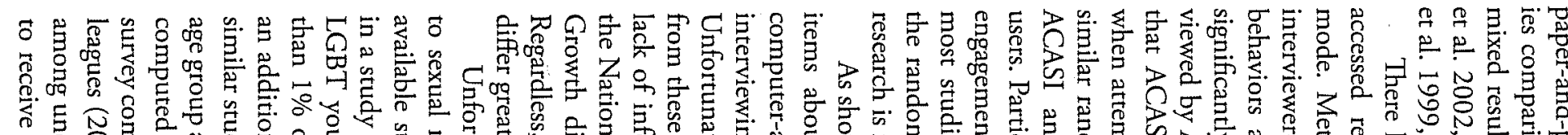

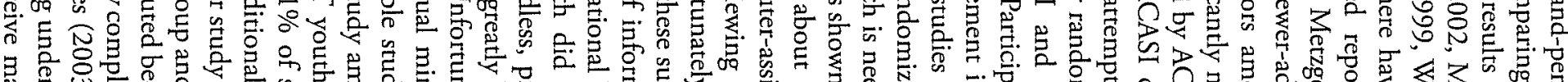

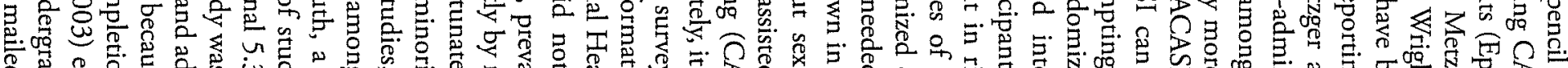

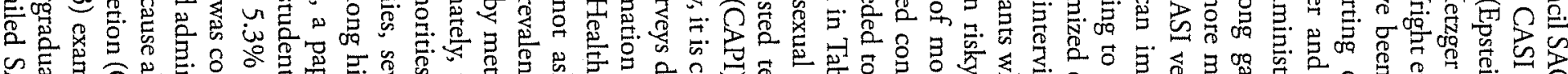

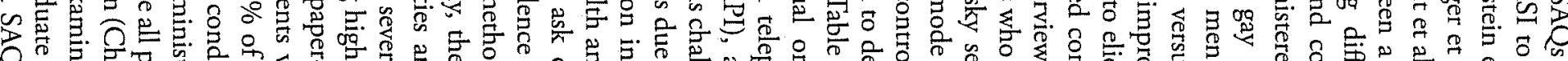
等

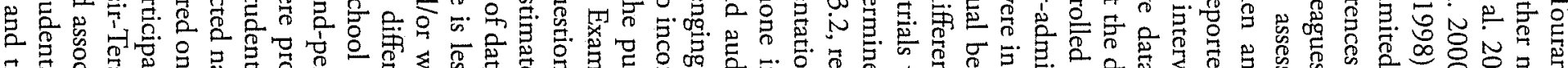

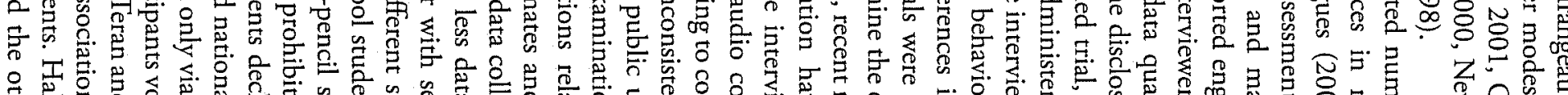

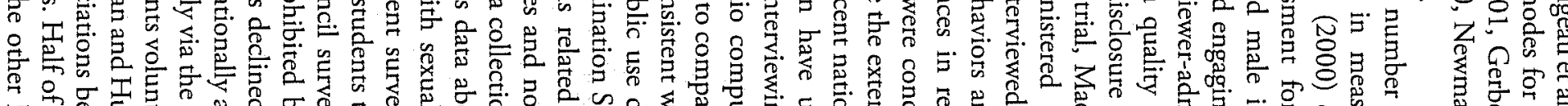

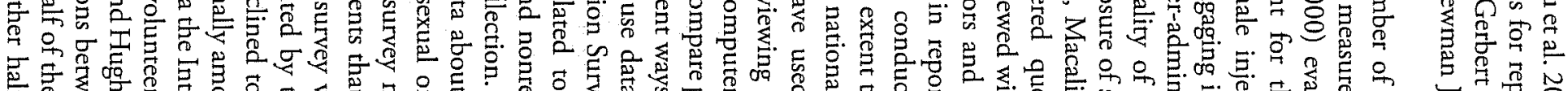

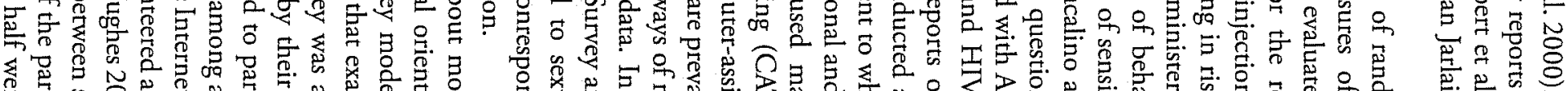

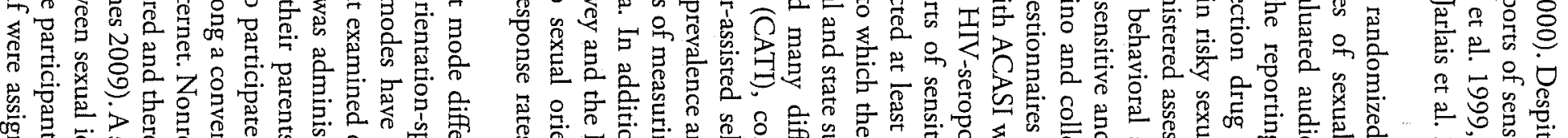

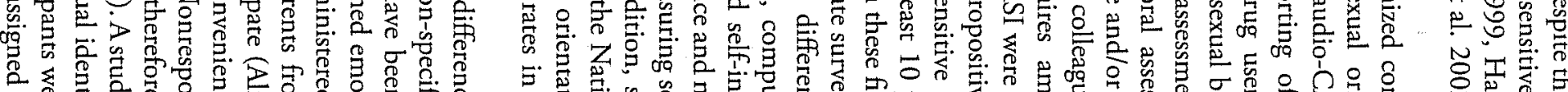

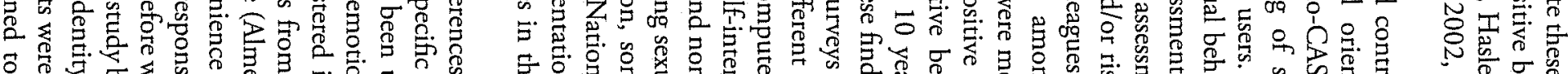

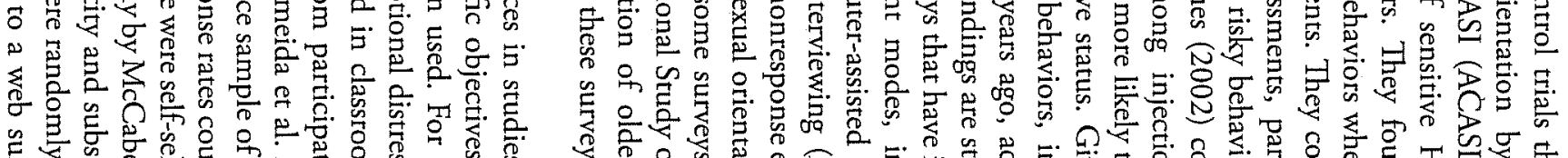

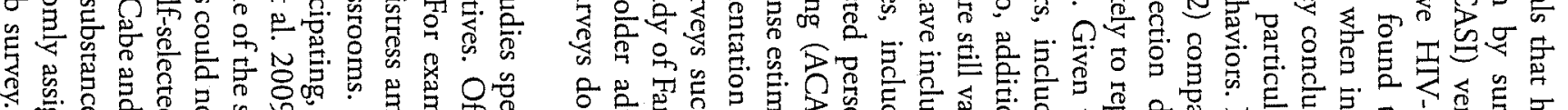

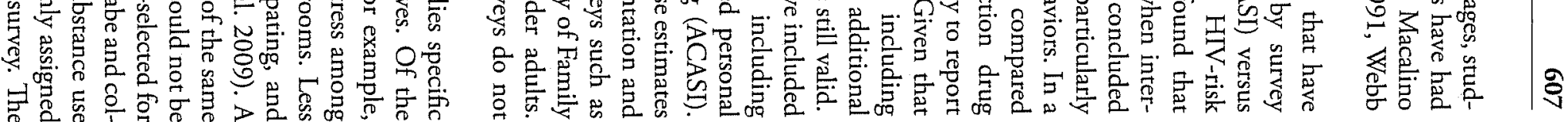




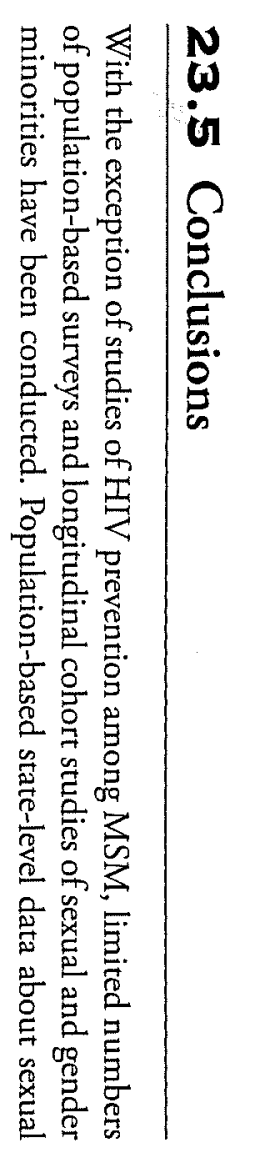

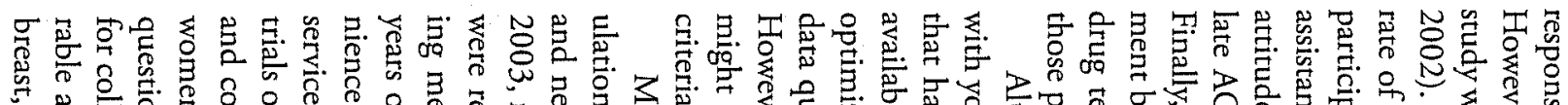

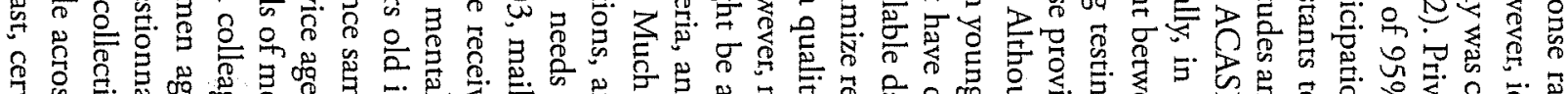

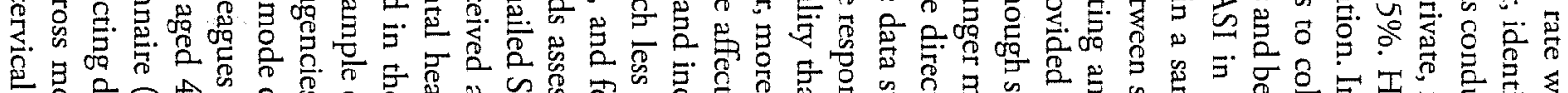

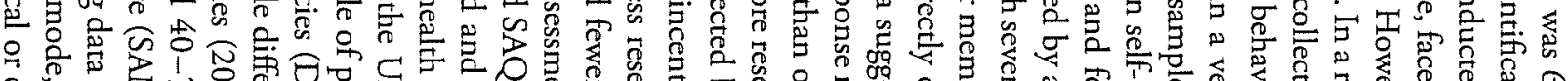

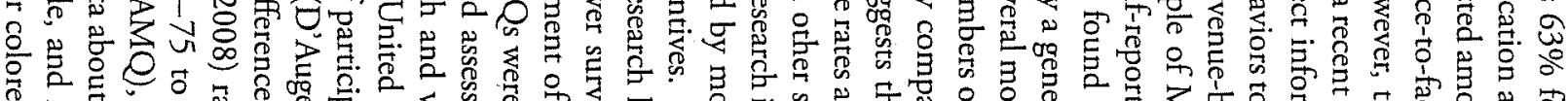

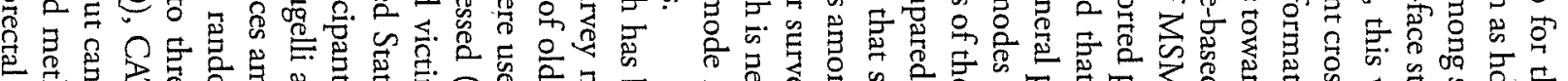

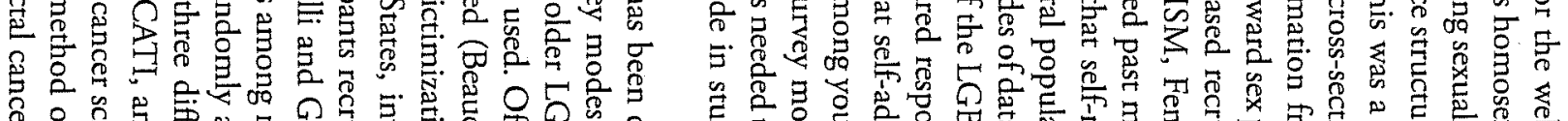

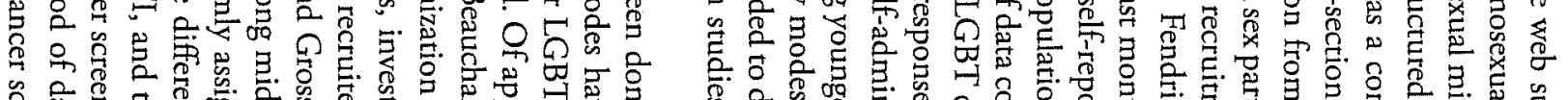

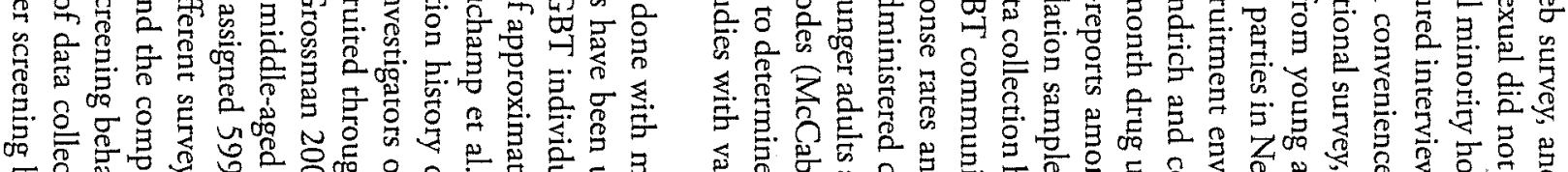

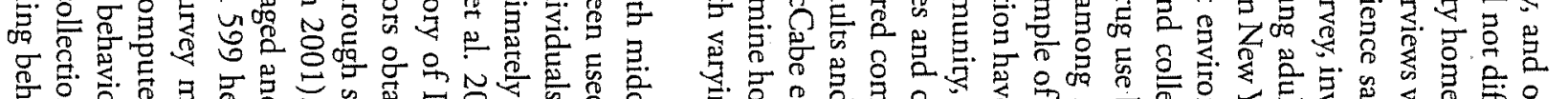
等

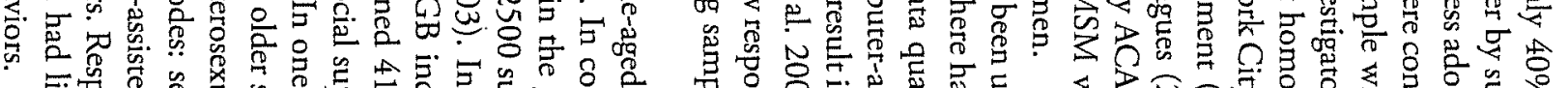

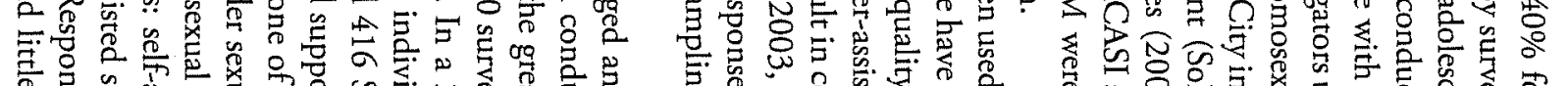

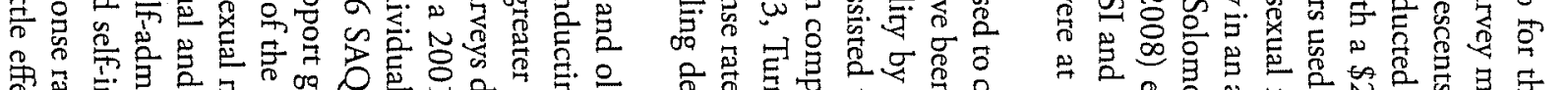

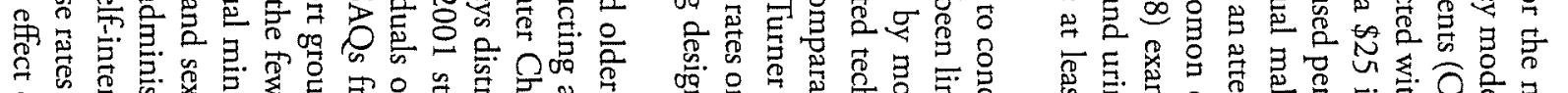

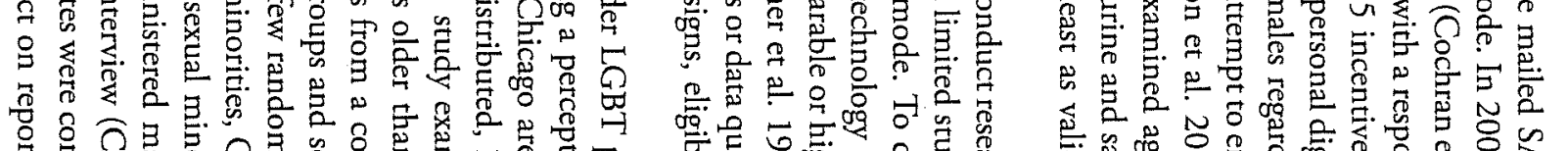

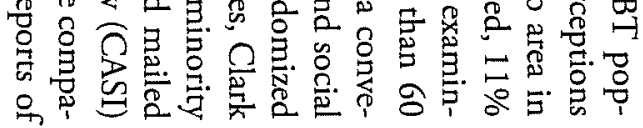

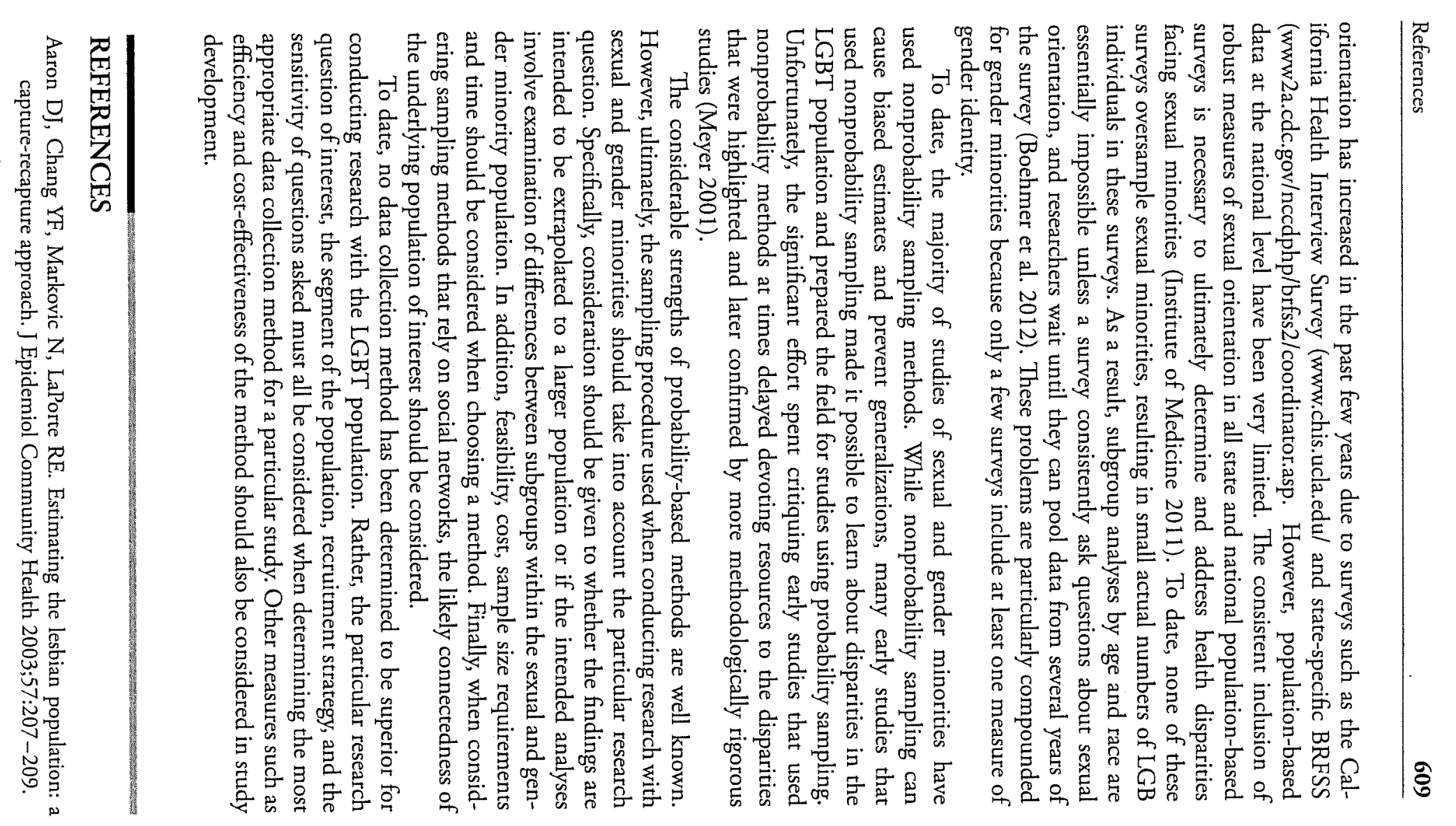




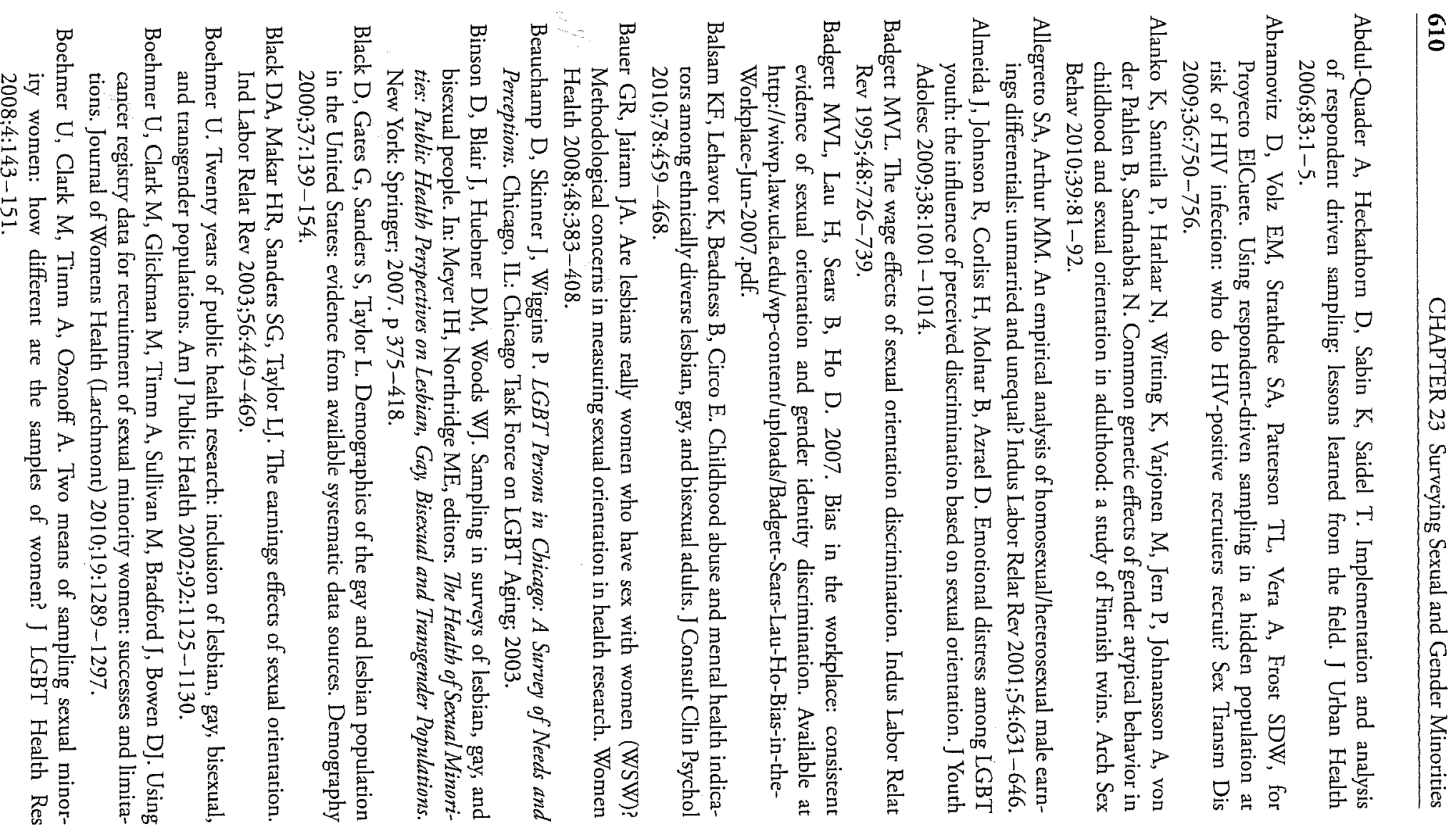

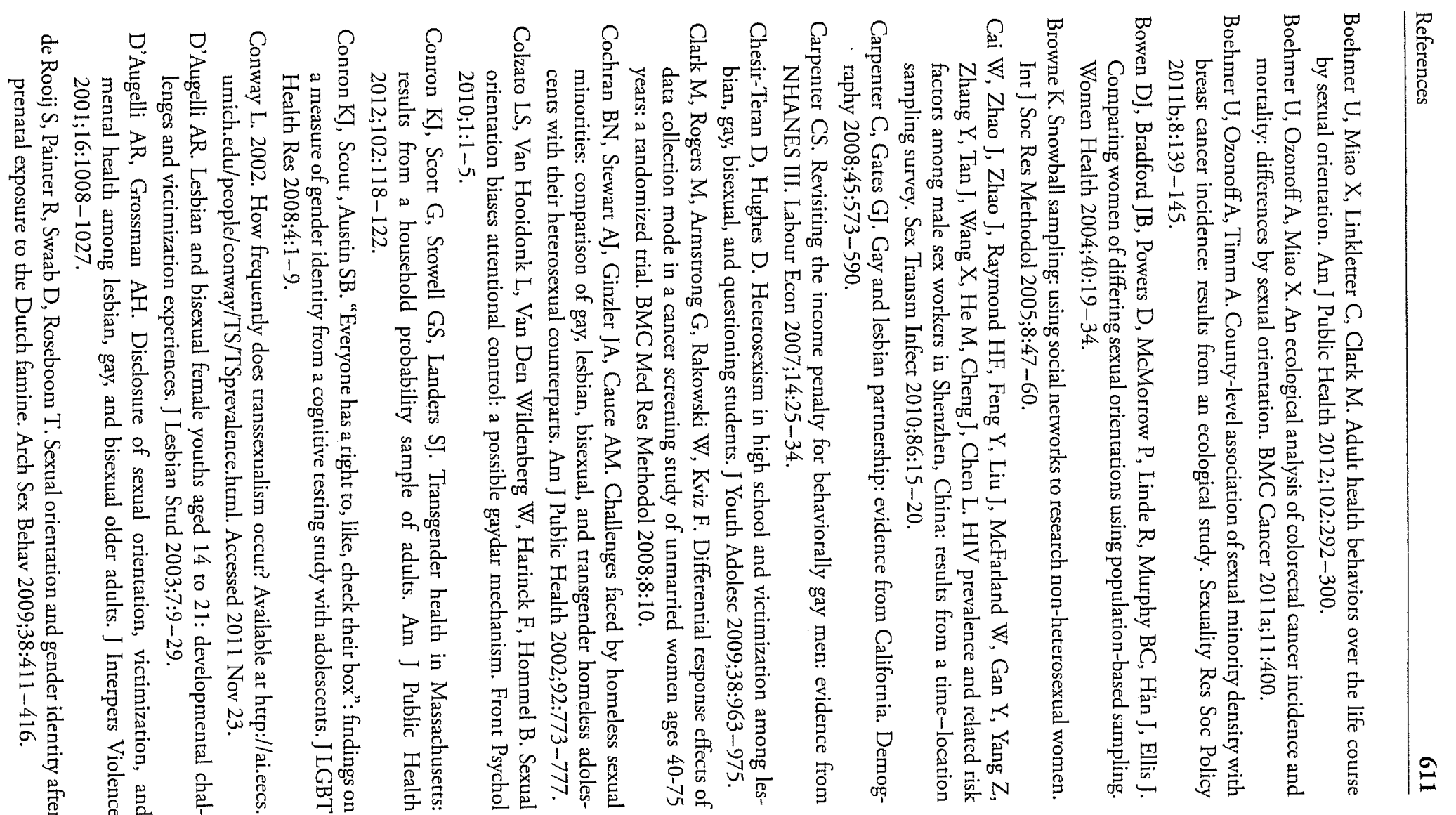




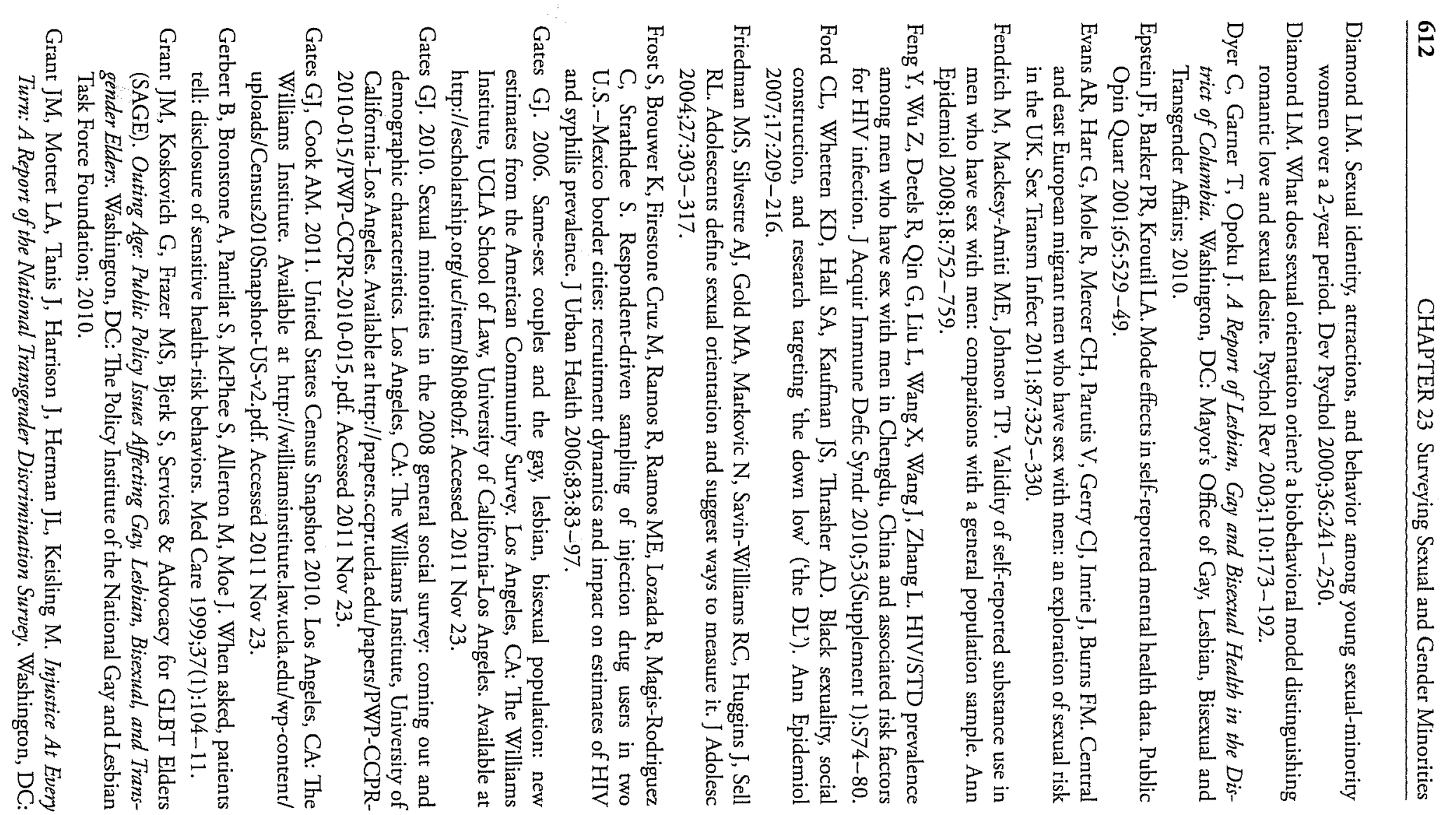

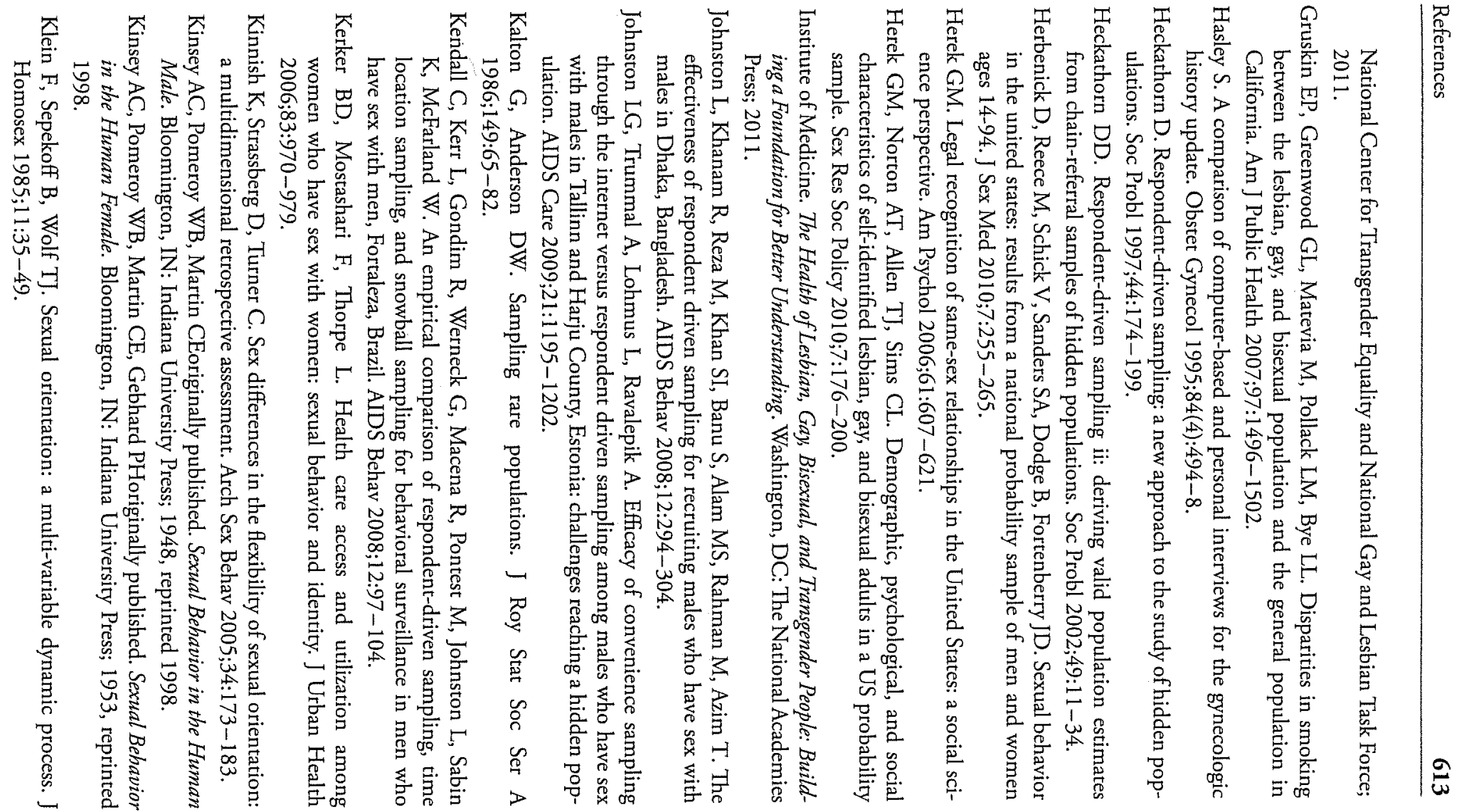




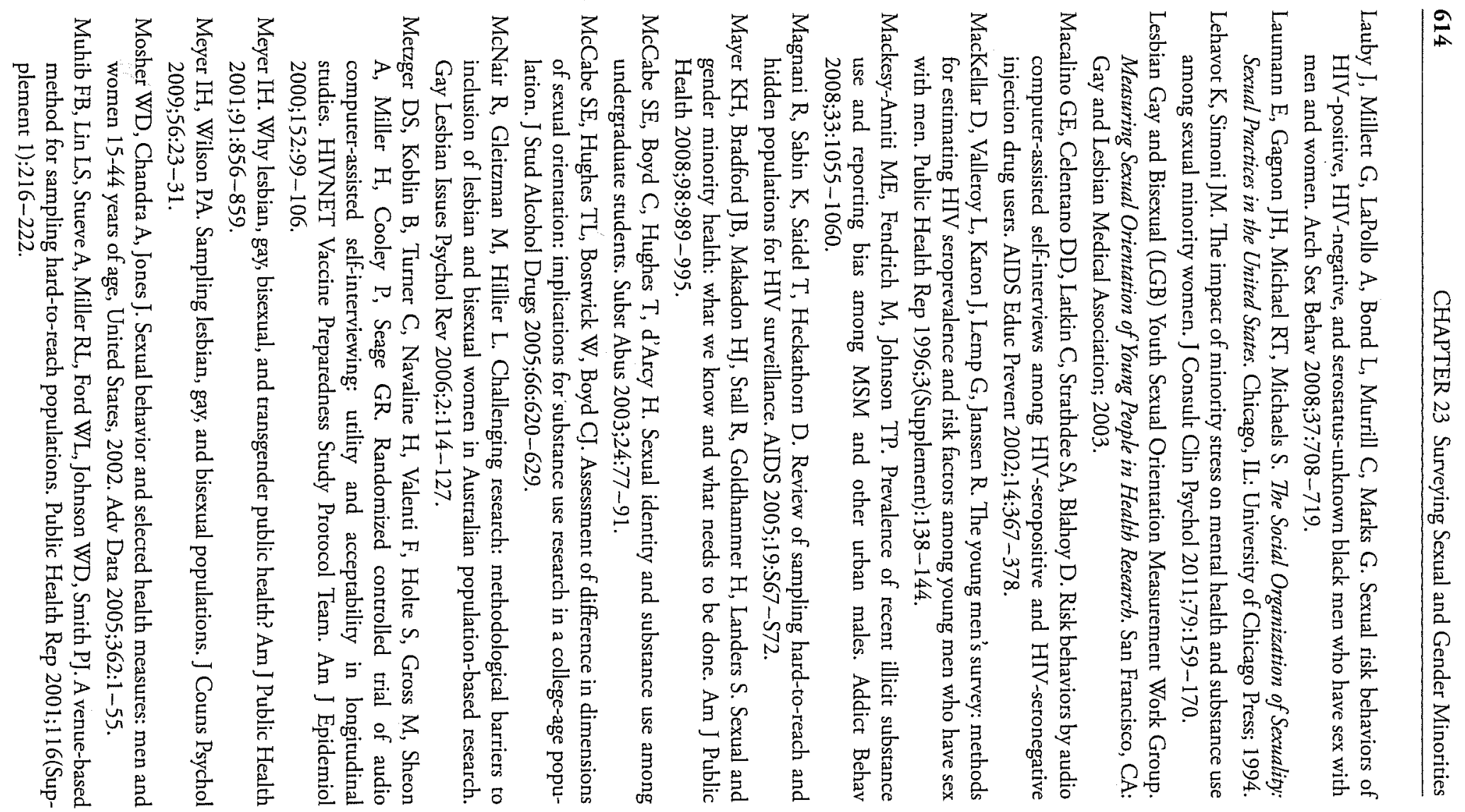

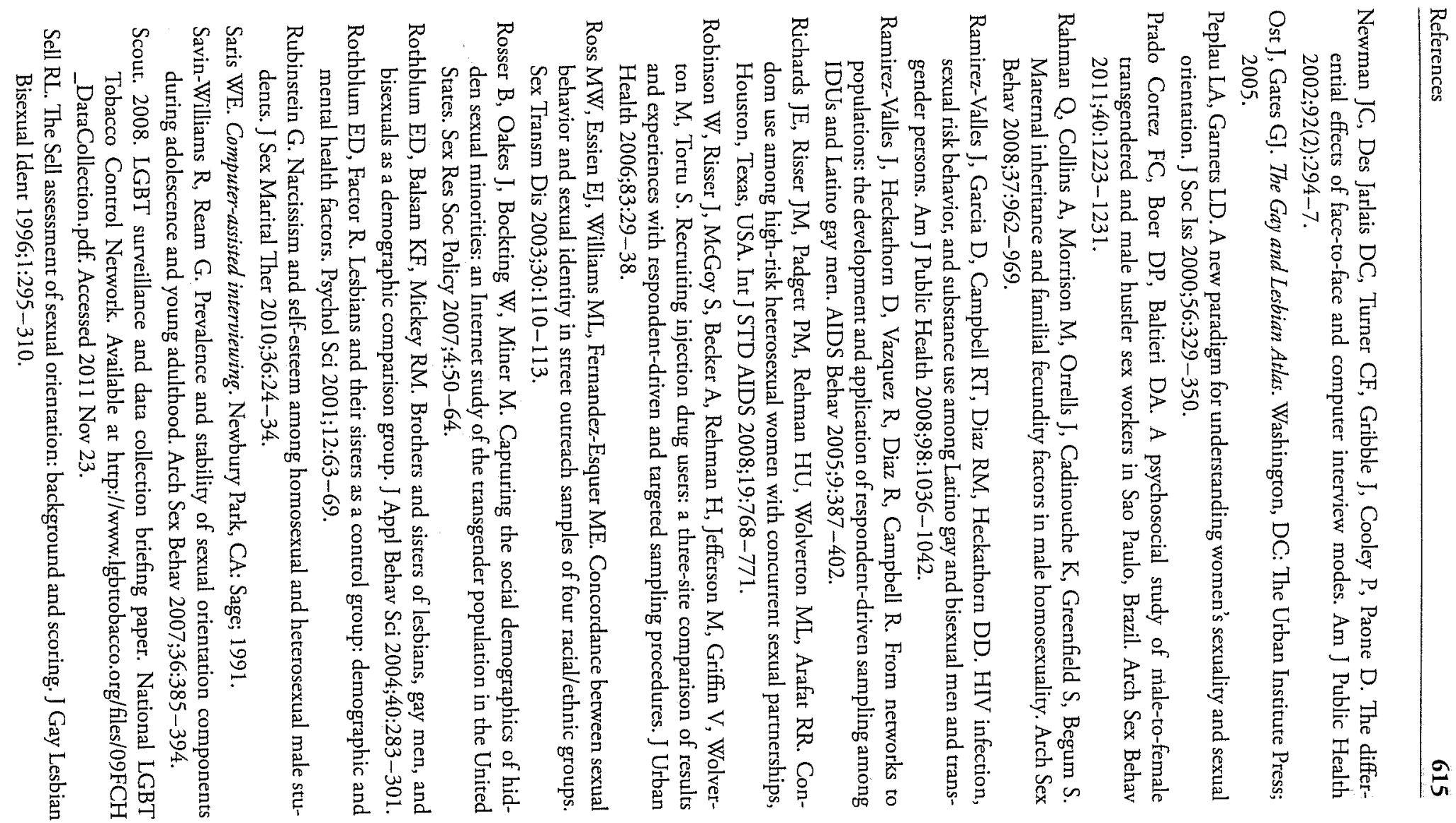


